\title{
Improving active Mealy machine learning for protocol conformance testing
}

\author{
Fides Aarts · Harco Kuppens · Jan Tretmans • \\ Frits Vaandrager $\cdot$ Sicco Verwer
}

Received: 17 December 2012 / Accepted: 7 August 2013 / Published online: 3 October 2013

(C) The Author(s) 2013

\begin{abstract}
Using a well-known industrial case study from the verification literature, the bounded retransmission protocol, we show how active learning can be used to establish the correctness of protocol implementation $I$ relative to a given reference implementation $R$. Using active learning, we learn a model $M_{R}$ of reference implementation $R$, which serves as input for a model-based testing tool that checks conformance of implementation $I$ to $M_{R}$. In addition, we also explore an alternative approach in which we learn a model $M_{I}$ of implementation $I$, which is compared to model $M_{R}$ using an equivalence checker. Our work uses a unique combination of software tools for model construction (Uppaal), active learning (LearnLib, Tomte), model-based testing (JTorX, TorXakis) and verification (CADP, MRMC). We show how these tools can be used for learning models of and revealing errors in implementations, present the new notion of a conformance oracle, and demonstrate how conformance oracles can be used to speed up conformance checking.
\end{abstract}

Keywords Active learning · Automaton learning $\cdot$ Mealy machines $\cdot$ State machine synthesis $\cdot$ Model-based testing $\cdot$ Protocol learning $\cdot$ Model checking

\section{Introduction}

Active learning is a type of machine learning in which the learner (an algorithm) is allowed to ask questions (queries) to an oracle, see, e.g. Settles (2010). In machine learning, such an

Editors: Jeffrey Heinz, Colin de la Higuera, and Tim Oates.

F. Aarts $\cdot$ H. Kuppens $\cdot$ J. Tretmans $\cdot$ F. Vaandrager $\cdot$ S. Verwer $(\varangle)$

Institute for Computing and Information Sciences, Radboud University Nijmegen, P.O. Box 9010, 6500

GL Nijmegen, The Netherlands

e-mail: siccoverwer@gmail.com

J. Tretmans

TNO_Embedded Systems Innovation, P.O. Box 513, 5600 MB Eindhoven, The Netherlands 
oracle is frequently seen as a human annotator which can assign labels to training instances. A learner can use this feedback to find or improve a model for the training data. Moreover, by asking informative queries (e.g., close to the decision boundary), an active learner potentially requires fewer examples than a passive learner that learns from data samples. Since annotation can be quite costly, the goal is to ask as few questions as possible in order to reach a good quality model.

This paper is concerned with an application of active learning. However, instead of asking queries to a human annotator, we use and develop methods that ask queries to software systems. These methods provide a software system with input (a training instance), read the corresponding output (e.g., a label), and use this to update their current hypothesis (model) of the software's internal behavior. Although the goal in both of these settings is to learn a good model, there exists a fundamental difference in the cost associated with asking queries. In the case of software systems, this cost is (usually) low. Our methods can consequently ask millions of queries within minutes. The LearnLib toolkit (Raffelt et al. 2009), winner of the 2010 Zulu active state machine learning competition (Combe et al. 2010), can therefore learn models consisting of up to tens of thousands of states. Active state machine learning tools have been used successfully for many different applications in software engineering such as regression testing of software components (Hungar et al. 2003), fuzz testing of protocol implementations (Comparetti et al. 2009), and inference of botnet protocols (Cho et al. 2010).

Many active state machine learning algorithms are based on the well-known $L^{*}$-algorithm (Angluin 1987). In addition to asking membership queries (input-output pairs), $L^{*}$ asks equivalence queries, which test whether the current hypothesis is correct. Intuitively, the membership queries are used to single out a state machine $\mathcal{M}$ within a certain size bound by proving all others to be inconsistent, an equivalence query is then used to find a counterexample for $\mathcal{M}$ and increase this size bound. $L^{*}$ uses the information contained in these counterexamples in order to use at most a polynomial number of membership queries in the size of the learned model. While usually only a few counterexamples are needed for $L^{*}$ to converge, answering equivalence queries is often the bottleneck in active state machine learning application. The reason is that testing whether a black-box software system behaves according to a given state machine description is hard (Dalal et al. 1999). Constructing informative test queries (i.e., membership queries) is known as test selection and is one of the main problems dealt with in model-based software testing (Lee and Yannakakis 1996). Several software testing methods and tools have been developed for this task in order to aid software development and maintenance, see, e.g., Belinfante (2010), Mostowski et al. (2009), Shafique and Labiche (2010). Although, these methods are able to approximate equivalence queries by (smartly) asking many membership queries, an exponential amount of them (or one of exponential length) are required in the worst case.

In this paper, we investigate a novel application domain for active learning of software models: establishing the correctness of protocol implementations relative to a given reference implementation. In protocol engineering, protocols are typically specified by independent, authorative agencies, e.g., the ITU (International Telecommunication Union) or the IETF (Internet Engineering Task Force). For such a protocol specification, a reference implementation is then often developed, which helps to discover errors and ambiguities in the specification, demonstrates that the specification is actually implementable, and serves as a definite interpretation of the specification. Based on the specification and the reference implementation, independent suppliers will develop products implementing the protocol. The reference implementation is then the standard against which these products are measured and compared to assess compliance with the specification. 
To the best of our knowledge, this is a novel application area of grammatical inference and machine learning. Moreover, it is a promising one since reference implementations are in existence for many real-world software systems, but models are usually lacking or incomplete (Aarts et al. 2010a, 2010b).

Our investigation is focused on a well-known benchmark case study from the verification literature: the bounded retransmission protocol (Helmink et al. 1994; D'Argenio et al. 1997) (see Sect. 5). The bounded retransmission protocol is a variation of the classical alternating bit protocol (Bartlett et al. 1969) that was developed by Philips Research to support infrared communication between a remote control and a television. We constructed an implementation of the protocol, to which we refer as the reference implementation, and 6 other faulty variations of the reference implementation (see Appendix A). Our aim is to combine active learning methods with model-based testing (see Sect. 3) in order to quickly discover the behavioral differences between these variations and the reference. To this aim, we make use of several state-of-the-art tools from grammatical inference, software testing, and formal verification (see Sect. 3 and Appendix B). We show how these tools can be used for learning models of the bounded retransmission protocol and revealing implementation errors in the mutants (Sects. 6 and 7).

In addition to experimental results on learning the bounded retransmission protocol, we provide two solutions that significantly reduce the difficulty of answering equivalence queries in this setting:

1. Using abstractions over input and output values through our Tomte learning tool (Aarts et al. 2012a) and through the model-based testing tool TorXakis (Mostowski et al. 2009) (see Sect. 3). Tomte performs on-the-fly abstractions on concrete input and output values, only introducing new abstract values when required for learning.

2. Using a previously learned model of the reference implementation together with the current hypothesis in a model equivalence checker (such as the popular CADP model checker (Garavel et al. 2011)). The resulting agent, which we call a conformance oracle (see Sect. 4), effectively transfers knowledge from the reference learning task to the task of learning a mutant, i.e., a slight variation of the reference implementation. The speedup results from the fact that test selection is difficult while equivalence testing is easy.

Our main contributions are demonstrating how active learning can be used in an industrial setting by combining it with software verification and testing tools, and showing how these tools can also be used to analyze and improve the results of learning. The bounded retransmission protocol use case can serve as a benchmark for future active learning and testing methods.

Our research takes place at the interface of model-based testing and model inference, and builds upon a rich research tradition in this area. The idea of combining testing and learning of software systems was first explored by Weyuker, who observed in 1983 "Program testing and program inference can be thought of as being inverse processes" (Weyuker 1983). Recently, there has been much interest in relating model-based testing and model inference in the setting of state machines. Berg et al. (2005), for instance, point out that some of the key algorithms that are used in the two areas are closely related. Walkinshaw et al. (2010b) show that active learning itself is an important source of structural test cases. At the ISoLA 2012 conference a special session was dedicated to the combination of model-based testing and model inference (Meinke and Walkinshaw 2012), a combination which is often denoted by the term learning-based testing. As far as we know, no previous work in this area addresses the problem of conformance with respect to a reference implementation. In addition, the specific combination of tools that we use is new, as well as the case study, and the concept of a conformance oracle. 
A preliminary version of this paper appeared as Aarts et al. (2012b). Besides providing background information, technical details, and full/improved experimental data, the most significant contribution beyond Aarts et al. (2012b) is the new notion of a conformance oracle.

\section{Software model synthesis}

The motivation of our work stems from the fact that the behavior of software systems can often be specified using finite state machine models. These models capture the behavior of software systems, by describing the way in which they react to different inputs, and when they produce which output. Visualizing such state machines can provide insights into the behavior of a software system, which can be of vital importance during the design and specification of a system. Moreover, state machines can be used to test and analyze a software system's properties using model checking (Clarke 1997) and testing techniques (Broy et al. 2005). In practice, one often encounters software systems without formal behavioral specifications. Reasons for this situation are manyfold: developing and maintaining them is often considered to be too costly (Walkinshaw et al. 2010a), legacy software is often kept running while its documentation is lost or outdated, computer viruses are specifically designed to be difficult to comprehend, and existing software models are often proprietary information of the software developer.

An alternative to constructing these models manually, is to use software model synthesis (or system identification/learning, or process discovery/mining) tools in order to derive them automatically from data (Cook and Wolf 1998). Software model synthesis is a technique for automatically constructing a software model based on observed system behavior. This data typically consists of execution traces, i.e., sequences of operations, function calls, user interactions, or protocol primitives, which are produced by the system or its surrounding environment. Intuitively, software model synthesis tries to discover the logical structure (or model) underlying these sequences of events. This can be seen as a grammatical inference problem in which the events are modeled as the symbols of a language, and the goal is to find a model for this language. The problem of learning state machines therefore enjoys a lot of interest from the software engineering and formal methods communities. Many different language models and ways of finding them are available in the grammatical inference (e.g., de la Higuera 2010) literature. Which one to choose depends mostly on the available data and the type of system under consideration.

In the context of this paper, we assume the existence of a reference implementation of a well-known benchmark case study from the verification literature. Given the reference implementation and some possibly faulty implementations provided by suppliers, our goal is to quickly discover the behavioral differences between the reference and the supplied programs. Using a reference implementation in order to establish the correctness of other implementations is an important problem in software engineering since reference implementations are in existence for many real-world software systems. Furthermore, since models are usually lacking or incomplete, it is often impossible to verify correctness or other properties using existing verification technologies such as model checking.

The models that we use in this paper are Mealy machines, which are a deterministic finite state automaton (DFA) variant with alternating input and output symbols, see, e.g., Sudkamp (2006). Mealy machines are popular for specifying the behavior of reactive systems and communication protocols. DFAs and Mealy machines are simple models and in some 
cases they will not be able to represent or identify all the complex behaviors of a software system. Some more powerful models with learning algorithms include: non-deterministic automata (Yokomori 1993; Denis et al. 2000), probabilistic automata (Clark and Thollard 2004; Castro and Gavaldà 2008), Petri-nets (van der Aalst 2011), timed automata (Verwer 2010; Verwer et al. 2011; Grinchtein et al. 2006), I/O automata (Aarts and Vaandrager 2010), and Büchi automata (de la Higuera and Janodet 2004). Despite their limited power, DFA and Mealy machine learning methods have recently been applied successfully to learn different types of complex systems such as web-services (Bertolino et al. 2009), X11 windowing programs (Ammons et al. 2002), network protocols (Cui et al. 2007; Antunes et al. 2011; Comparetti et al. 2009), and Java programs (Walkinshaw et al. 2007; Dallmeier et al. 2006; Mariani et al. 2011).

We expect that the approaches that are explored in this paper for Mealy machines can also be applied in the setting of more powerful modeling frameworks. In Aarts and Vaandrager (2010), for instance, we have shown that the specific restriction of Mealy machines that inputs and outputs have to alternate, which is often inconvenient in practice, is not fundamental: any tool for active learning of Mealy machines can be used for learning I/O automata that are deterministic and output determined.

\section{Mealy machines, active learning, and model-based testing}

We learn Mealy machines from queries using tools that build upon the well-known $L^{*}$ algorithm, see Angluin (1987), Kearns and Vazirani (1994). In query learning, access to an oracle is needed that can answer specific types of questions such as: whether a specific string is part of the language (membership queries), and whether a given model is a model for the language (equivalence queries). In software model synthesis, the actual software system can be used for this purpose, see, e.g., Raffelt et al. (2009). When such an oracle is available that can be queried often and quickly, it is possible to identify very large realistic models. In this section, we first give formal descriptions of Mealy machines and the methods for testing and learning them. Afterwards, we describe how we combine state-of-the-art testing and learning tools in order to establish the conformance of an implementation relative to a given reference implementation.

\subsection{Mealy machines}

Mealy machines are a type of transducer very similar to deterministic finite state automata (DFAs, see, e.g., Sudkamp 2006). Instead of accepting or rejecting (classifying) an input string, Mealy machines produce (transduce) an output symbol for every input symbol transition visited (fired) by this run. Since software systems typically produce multiple outputs for multiple inputs, this makes them very suitable as software models. In particular, Mealy machines are very popular models for communication protocols since these often alternate between input and output communication.

Definition 1 A Mealy machine $(M M)$ is a tuple $\mathcal{M}=\left\langle I, O, Q, q_{0}, \rightarrow\right\rangle$, where

- I, O, and $Q$ are nonempty sets of input symbols, output symbols, and states, respectively,

$-q_{0} \in Q$ is the initial state, and

- $\rightarrow \subseteq Q \times I \times O \times Q$ is the transition relation.

We write $q \stackrel{i / o}{\longrightarrow} q^{\prime}$ if $\left(q, i, o, q^{\prime}\right) \in \rightarrow$. 


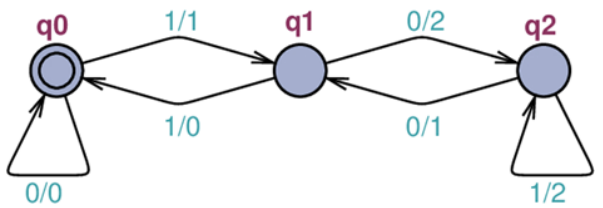

Fig. 1 A Mealy machine for computing residues modulo 3. For example, the machine maps 010 to 012 (path: $q_{0} \stackrel{0 / 0}{\longrightarrow} q_{0}, q_{0} \stackrel{1 / 1}{\longrightarrow} q_{1}, q_{1} \stackrel{0 / 2}{\longrightarrow} q_{2}$ ) computing $0 \bmod 3=0,1 \bmod 3=1,2 \bmod 3=2 ; 1101$ is mapped to 1001 computing $1 \bmod 3=1,3 \bmod 3=0,6 \bmod 3=0,13 \bmod 3=1 ;$ and 1110 to 1012 computing $1 \bmod 3=1,3 \bmod 3=0,7 \bmod 3=1,14 \bmod 3=2$

Table 1 Transition relation for the Mealy machine in Fig. 1

\begin{tabular}{llllll}
\hline Source state & \multicolumn{2}{l}{ Input } & & & \\
\cline { 2 - 3 } \cline { 5 - 6 } & Output & Target state & & Output & Target state \\
\hline$q_{0}$ & 0 & $q_{0}$ & 1 & $q_{1}$ \\
$q_{1}$ & 2 & $q_{2}$ & 0 & $q_{0}$ \\
$q_{2}$ & 1 & $q-1$ & 2 & $q_{2}$ \\
\hline
\end{tabular}

Mealy machines are assumed to be input enabled (or completely specified): for each state $q$ and input $i$, there exists an output $o$ such that $q \stackrel{i / o}{\longrightarrow}$. An intuitive interpretation of a Mealy machine is as follows. At any point in time, the machine is in some state $q \in Q$. It is possible to give inputs to the machine by supplying an input symbol $i \in I$. The machine then selects a transition $q \stackrel{i / o}{\longrightarrow} q^{\prime}$, produces output symbol $o$, and jumps to the new state $q^{\prime}$. A Mealy machine $\mathcal{M}$ is deterministic if for each state $q$ and each input symbol $i$ there is exactly one output symbol $o$ and exactly one state $q^{\prime}$ such that $q \stackrel{i / o}{\longrightarrow} q^{\prime}\left(q, q^{\prime} \in Q, i \in I\right)$. We say that a Mealy machine is finite if the set $Q$ of states and the set $I$ of inputs are finite.

Example 1 An example of a Mealy machine for computing residues modulo 3 for a binary input (most significant bit first) number is given in Fig. 1. Given an input word $i_{1} \ldots i_{n}$, the $j$ th output of the machine corresponds to $i_{1} \ldots i_{j}$ modulo 3 . The set of inputs is $I=\{0,1\}$, the set of outputs is $O=\{0,1,2\}$, and the set of states is given by $Q=\left\{q_{0}, q_{1}, q_{2}\right\}$, where $q_{0}$ is the initial state marked with an extra circle. The transition relation is defined by Table 1.

The transition relation in Mealy machines is extended from symbols to strings (traces, sequences) as follows:

Definition 2 The extended transition relation $\stackrel{u / s}{\Rightarrow}$ on a Mealy machine $\mathcal{M}=\left\langle I, O, Q, q_{0}, \rightarrow\right\rangle$ is the least relation that satisfies, for $q, q^{\prime}, q^{\prime \prime} \in Q, u \in I^{*}, s \in O^{*}, i \in I$, and $o \in O$ :

$-q \stackrel{\epsilon / \epsilon}{\Rightarrow} q$, and

- if $q \stackrel{i / o}{\longrightarrow} q^{\prime}$ and $q^{\prime} \stackrel{u / s}{\Rightarrow} q^{\prime \prime}$ then $q^{i} \stackrel{\text { u/os }}{\Rightarrow} q^{\prime \prime}$,

where $\epsilon$ denotes the empty sequence. A state $q \in Q$ is called reachable if $q_{0} \stackrel{u / s}{\Rightarrow} q$, for some $u \in I^{*}$ and $s \in O^{*}$. 
The output strings that can be observed after supplying an input string to a Mealy machine are defined using this relation:

Definition 3 An observation is a pair $(u, s) \in I^{*} \times O^{*}$ such that sequences $u$ and $s$ have the same length. For $q \in Q$, we define $\operatorname{obs}_{\mathcal{M}}(q)$ to be the set of observations of $\mathcal{M}$ from state $q$, by

$$
o b s_{\mathcal{M}}(q)=\left\{(u, s) \in I^{*} \times O^{*} \mid \exists q^{\prime}: q \stackrel{u / s}{\Rightarrow} q^{\prime}\right\} .
$$

We write $o b s_{\mathcal{M}}$ as a shorthand for $o b s_{\mathcal{M}}\left(q_{0}\right)$.

Since Mealy machines are input enabled, $o b s_{\mathcal{M}}(q)$ contains at least one pair $(u, s)$ for each input sequence $u \in I^{*}$. We call $\mathcal{M}$ behavior deterministic if $o b s_{\mathcal{M}}$ contains exactly one pair $(u, s)$, for each $u \in I^{*}$. In this case, we write $o u t_{\mathcal{M}}(u)$ to denote the unique $s$ with $(u, s) \in o b s_{\mathcal{M}}$. In this paper, we consider only behavior deterministic Mealy machines. It is easy to see that a deterministic Mealy machine is also behavior deterministic. Two states $q, q^{\prime} \in Q$ are observation equivalent, denoted $q \approx q^{\prime}$, if $o b s_{\mathcal{M}}(q)=o b s_{\mathcal{M}}\left(q^{\prime}\right)$. Two Mealy machines $\mathcal{M}_{1}$ and $\mathcal{M}_{2}$ with the same sets of input symbols are observation equivalent, notation $\mathcal{M}_{1} \approx \mathcal{M}_{2}$, if $o b s_{\mathcal{M}_{1}}=o b s_{\mathcal{M}_{2}}$.

\subsection{Active learning of Mealy machines}

Active learning or query learning is a learning setting in which a learner can ask questions (queries) to a teacher. In our case, the teacher consists of an implementation, a black-box software system that we would like to analyze, in combination with an oracle that produces statements about the correctness of models produced by the learner. By providing the implementation with inputs, and reading the generated outputs, the learner tries to determine (reverse engineer) its inner workings. Once it has constructed a hypothesis (a model) for these workings, it asks the oracle whether this hypothesis is correct. With some modifications (Margaria et al. 2004), we can apply the well-known $L^{*}$ DFA learning algorithm (Angluin 1987) to this setting in order to learn a Mealy machine model for a black-box software system. The basic setup for active learning is illustrated in Fig. 2.

Let $\mathcal{M}=\left\langle I, O, Q, q_{0}, \rightarrow\right\rangle$ be a behavior deterministic Mealy machine. An implementation of $\mathcal{M}$ is a device that accepts sequences $u \in I^{*}$ of input symbols, called output queries or membership queries. Whenever an implementation of $\mathcal{M}$ receives a query $u$, it returns out $_{\mathcal{M}}(u)$ as output.

An oracle is a device which accepts Mealy machine models as inputs. These input models are referred to as equivalence queries or hypotheses. Upon receiving a hypothesis $\mathcal{H}$, the oracle may experiment with an implementation of a Mealy machine $\mathcal{M}$ by posing output queries. After a finite number of output queries, the oracle will either produce output pass together with model $\mathcal{H}$, or else output fail together with a counterexample, which is an observation $(u, s) \in\left(o b s_{\mathcal{M}} \backslash o b s_{\mathcal{H}}\right) \cup\left(o b s_{\mathcal{H}} \backslash o b s_{\mathcal{M}}\right)$. A proper oracle is an oracle that only generates pass when $\mathcal{M} \approx \mathcal{H}$. The combination of an implementation of $\mathcal{M}$ and a proper oracle corresponds to what Angluin (Angluin 1987) calls a minimally adequate teacher for $\mathcal{M}$.

A learner is a device that, once it is triggered by a go-command, may pose output queries to an implementation of some (unknown) Mealy machine $\mathcal{M}$, and equivalence queries to an oracle for this implementation, see Fig. 2. The task of the learner is to learn a model that is observation equivalent to $\mathcal{M}$ in a finite number of steps, from the answers generated by 


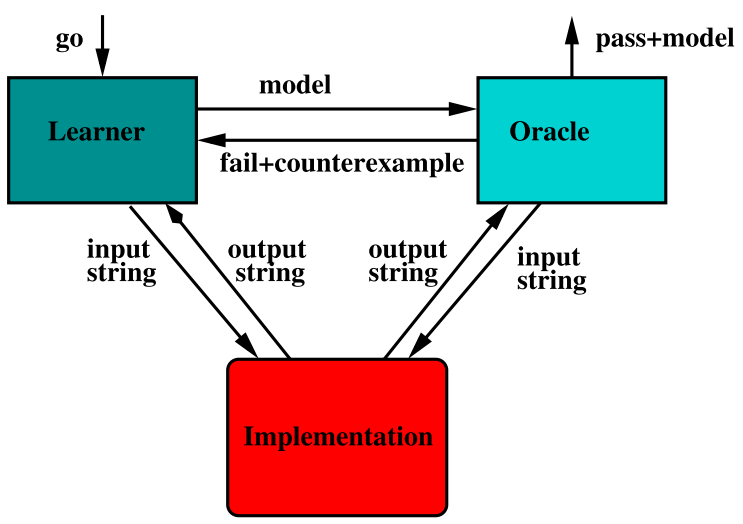

Fig. 2 Basic setting for active learning of software models. A learner starts by providing input sequences to an implementation of the software system (output queries), the implementation produces the corresponding outputs. By intelligently choosing these inputs, the learner is able to form a hypothesis model of the implementation's internal behavior. This model is provided to an oracle (equivalence queries). The oracle then tests (by providing inputs and checking the generated outputs) whether this model correctly describes the inner workings of the implementation. If so, it returns pass. Otherwise, it returns fail and a counterexample demonstrating this incorrectness

the implementation and the oracle in response to the queries. The typical behavior of an $L^{*}$-style learner is to start by asking sequences of output queries until a "stable" hypothesis $\mathcal{H}$ can be built from the answers. After that an equivalence query is made to find out whether $\mathcal{H}$ is correct. If the oracle answers pass then the learner has succeeded. Otherwise the returned counterexample is added to the set of observations, and additional output queries are asked until the learner has singled out a new smallest stable hypothesis $\mathcal{H}^{\prime}$ that is consistent with the current set of observations. This new hypothesis $\mathcal{H}^{\prime}$ is sent to the oracle in another equivalence query, and a possible counterexample is then again used in output queries in order to update the current hypothesis. This process is iterated until the oracle answers pass in response to an equivalence query. A nice property of $L^{*}$ and query learning is that, assuming a proper oracle, it requires only a polynomial number of queries in order to find $\mathcal{H}$ such that $\mathcal{M} \approx \mathcal{H}$. This is surprising since learning the smallest deterministic finite state automaton (and by restriction Mealy machine) that is consistent with a given dataset is well-known to be NP-hard (Gold 1978).

LearnLib LearnLib (Raffelt et al. 2009) is a tool that supports active learning of Mealy machines or DFAs based on the $L^{*}$ algorithm. It contains many optimizations that reduce the number of queries asked by $L^{*}$, and a means of selecting them in a graphical user interface. Furthermore, it implements multiple learning strategies, that differ in the method used to construct the model (depth-first or breadth-first), and includes different ways to generate membership queries. Finally, the LearnLib tool also includes various model-based testing algorithms (see Sect. 3.4) in order to implement the oracle component. The LearnLib tool was used by the winning team in the 2010 Zulu DFA active learning competition (Combe et al. 2010). We use LearnLib as the basic active learning tool.

Uppaal The model-checker Uppaal (Behrmann et al. 2004) is one of the best known model checkers today. It is based on timed automata (Alur and Dill 1994) and can be used to test logical properties of these systems, specified using a subset of computation tree logic 


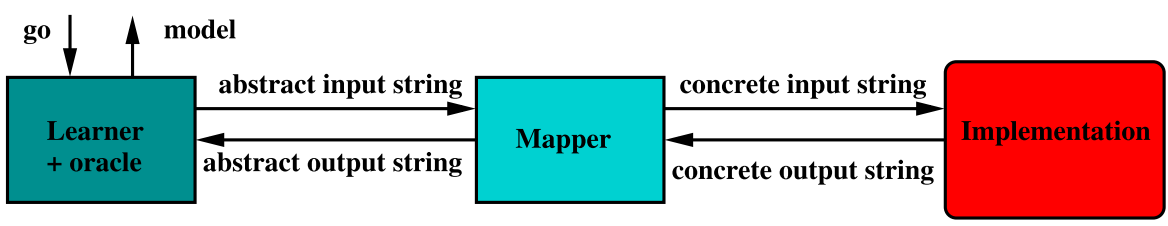

Fig. 3 Introduction of the mapper component. A mapper acts in between the implementation and the learner. It maps input sequences from the abstract (discrete) domain used by the learner to the concrete (integers/floats/messages) domain of the implementation. In reverse, it maps the concrete output to abstract output, which serves as input for the learner. An abstract input can be mapped to different concrete inputs and when two of these result in different abstract outputs, the mapper refines the abstraction on-the-fly in order to remove this non-deterministic behavior

(CTL) (Henzinger et al. 1994). In addition to the timed properties of systems, Uppaal models can contain integer variables, structured data types, and channel synchronizations between automata. It contains an extensive graphical user interface including an automaton editor, an intuitive query language, a simulator, and a verification engine.

In this article, we use the Uppaal GUI as an editor for extended finite state machines (EFSM).

\subsection{Automatic abstraction refinement}

Tools that are able to bridge the gap between active learning tools such as LearnLib and real software systems are required for numerous applications in different domains. Instead of learning deterministic finite state automata or Mealy machines, they aim at learning models of extended finite state machines (EFSM), including parameter values and guarded transitions based on these values. Abstraction is the key for scaling existing automata learning methods to realistic applications. The idea is to place a mapper in between the implementation and the learner/oracle, that abstracts (in a history dependent manner) the large set of actions of the implementation into a small set of abstract actions for the learner/oracle, see Fig. 3.

The concept of an intermediate component that takes care of abstraction is very natural and is used, implicitly or explicitly, in many case studies on automata learning. Aarts et al. (2010a) formalized the concept of such an intermediate abstraction component. By combining the abstract machine $\mathcal{H}$ learned in this way with information about the mapper, it is possible to effectively learn an EFSM that is equivalent to the Mealy machine $\mathcal{M}$ of the implementation. Roughly speaking, the learner is responsible for learning the global "control modes" in which the system can be, and the transitions between those modes, whereas the mapper records some relevant state variables (typically computed from the data parameters of previous input and output actions) and takes care of the data part of the implementation.

Tomte Aarts et al. (2012a) is an automatic abstraction refinement tool that uses and learns abstractions in order to map the extended finite state machine world of software systems into the Mealy machine world of active learning tools such as LearnLib. Tomte is named after the creature that shrank Nils Holgersson into a gnome and (after numerous adventures) changed him back to his normal size again. This is exactly what the Tomte tool is doing: It shrinks the alphabet in order to be able to learn an abstract model (through numerous experiments), and then the abstract model is enlarged again to a model of the original implementation by enriching it with information from the mapper component. 
The current version of Tomte is able to automatically construct mappers for a restricted class of extended finite state machines, which is called scalarset Mealy machines, in which one can test for equality of data parameters, but no operations on data are allowed. The notion of a scalarset data type originates from model checking, where it has been used by Ip $\&$ Dill for symmetry reduction (Ip and Dill 1996). Currently, Tomte can learn models that may only remember the first and last occurrence of a parameter. A major challenge is the development of new algorithms for the automatic construction of mappers: the availability of such algorithms will allow to infer a wider class of systems and boost the applicability of automata learning technology.

Tomte uses the technique of counterexample-guided abstraction refinement: initially, the algorithm starts with a very coarse abstraction, which is subsequently refined if it turns out that the learned model is not behavior-deterministic. Nondeterminism arises naturally when we apply abstraction: it may occur that the behavior of the implementation is fully deterministic but that due to the mapper (which, for instance, abstracts from the value of certain input parameters), the implementation appears to behave nondeterministically from the perspective of the learner. Tomte uses LearnLib as basic learning tool and therefore the abstraction of the implementation may not exhibit any nondeterminism: if it does then LearnLib crashes and Tomte has to refine the abstraction.

Using Tomte, it is possible to learn fully automatically models of several realistic software components, including the biometric passport (Aarts et al. 2010b) as well as the SIP and TCP protocol (Aarts et al. 2010a).

\subsection{Model-based testing}

Unfortunately, in practice there is unlikely to be an oracle that can answer equivalence queries, making a direct application of $L^{*}$ often impossible. The typical solution (also adopted in this paper) is to approximate these queries using randomly generated membership queries (Angluin 1987). Thus, for every equivalence query, we generate many input strings, for each we ask a membership query, and if an observed output string is different from the output generated by the model, we return this string as a counterexample. If no such string is found, there is some confidence that the current hypothesis is correct and the more strings we test, the higher this confidence. Since state machines cannot be learned from a polynomial amount of membership queries (Balcázar et al. 1997), this procedure requires an exponential number of queries in the worst case. Instead of generating these approximate equivalence queries uniformly at random, it therefore makes sense to use the current hypothesis and observations to only ask informative ones. The problem of finding such informative membership queries is known as test selection, which is one of the main problems dealt with by model-based testing.

In Model-based testing (MBT), which is a new technique that aims to make testing more efficient and more effective (Utting and Legeard 2007), the system under test (SUT) is tested against a model of its behavior. This model, which is usually developed manually, must specify what the SUT shall do. Test cases can then be algorithmically generated from this model using an MBT tool. When these test cases are executed on the SUT and the actual test outcomes are compared with the model, the result is an indication about compliance of the SUT with the model. Usually, MBT algorithms and tools are sound, i.e., a test failure assures non-compliance, but they are not exhaustive, i.e., absence of failing tests does not assure compliance: "Program testing can be used to show the presence of bugs, but never to show their absence!" (Dijkstra 1969).

MBT approaches differ in the kind of models that they support, e.g., state-based models, pre- and post-conditions, (timed) automata, or equational axioms, and in the algorithms that 
they use for test generation. In this paper we concentrate on two state-based approaches: finite, deterministic Mealy machines (also called Finite State Machine FSM), and a class of non-deterministic automata, also referred to as labeled transition systems (LTS).

In the Mealy machine approach to MBT, the goal is to test whether a black-box SUT, which is an implementation of an unknown Mealy machine $I$, is observation equivalent to a given Mealy machine specification $S$, i.e., to test whether $I \approx S$ (Lee and Yannakakis 1996).

The LTS approach, which does not require determinism, finiteness of states and inputs, input-enabledness, nor alternation of inputs and outputs (a label on a transition is either an input or an output), is more expressive than Mealy machines. Consequently, it requires a more sophisticated notion of compliance between an SUT and a model. The implementation relation ioco often serves this purpose (Tretmans 2008). The tools JTorX and TorXakis, among others, generate tests based on this relation; see below. Since it is straightforward to transform a Mealy machine into an LTS, by splitting every (input,output)-pair transition into two LTS transitions with an intermediate state, LTS-based testing can be easily applied to Mealy machine models.

JTorX JTorX (Belinfante 2010) is an update of the model-based testing tool TorX (Tretmans and Brinksma 2003). TorX is a model-based testing tool that uses labeled transition systems to derive and execute tests (execution traces) based on ioco (Tretmans 2008), a theory for defining when an implementation of a given specification is correct. Using on-line testing, TorX can easily generate and execute tests consisting of more than 1000000 test events. JTorX is easier to deploy and uses a more advanced version of ioco. It contains a graphical user interface for easy configuration, a simulator for guided evaluation of a test trace, interfaces for communication with an SUT, and state-of-the-art testing algorithms.

TorXakis TorXakis (Mostowski et al. 2009) is another extension of the TorX model-based testing tool. In addition to the testing algorithms, TorXakis uses Symbolic Transition Systems (STSs, LTSs with data variables) with symbolic test generation to deal with structured data, i.e., symbols with data parameters (Frantzen et al. 2005), where TorX and JTorX use flattening, i.e., by replacing a parameter by all its possible, necessarily finitely many values. By working symbolically and by exploiting the structure of input actions, TorXakis is able to find certain counterexamples much faster than LearnLib and JTorX.

\section{Conformance to a reference implementation}

\subsection{Conformance learning and conformance model-based testing}

Figure 4 illustrates how we may use model synthesis for establishing conformance (i.e., behavior equivalence) of protocol implementations relative to a given reference implementation. Using a state machine synthesis tool, we first actively (query) learn a state machine model $M_{R}$ of the reference implementation $R$ (using, e.g., LearnLib). Now, given another implementation $I$, there are basically two things we can do. The first approach, which we call 'conformance model-based testing', is that we provide $M_{R}$ as input to a model-based testing tool (e.g., JTorX). This tool will then use $M_{R}$ to generate test sequences and apply them to implementation $I$ in order to establish the conformance of $I$ to the learned model $M_{R}$, i.e., whether they implement the same behavior. The model-based testing tool will either output "pass", meaning that the tool has not been able to find any deviating behaviors, or it will output "fail" together with an input sequence that demonstrates the difference between 


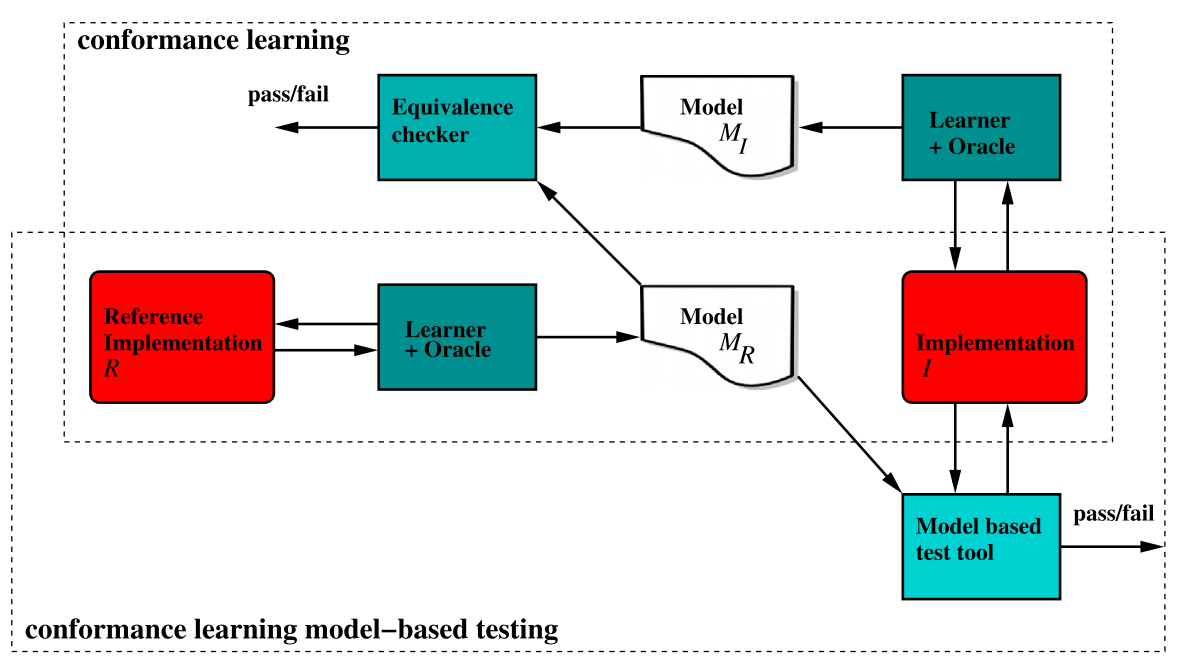

Fig. 4 Basic approaches of using automata learning to establish conformance of implementations. The learners interact with the implementations in order to construct models, which are then subsequently used for model-based testing or equivalence checking

$I$ and $M_{R}$. The second, more ambitious approach, which we call 'conformance learning', is to use the learning tool to learn a model $M_{I}$ of the other implementation $I$, and then use an equivalence checker to check observation equivalence of $M_{R}$ and $M_{I}$. The equivalence checker will either output "pass", meaning that the two models are equivalent, or "fail" together with an input sequence that demonstrates the difference between the two models. In the latter case, we check whether this trace also demonstrates a difference between the corresponding implementations $R$ and $I$. If not, we have obtained a counterexample for one of the two models, which we may feed to the learner in order to obtain a more refined model of $R$ or $I$.

In this paper, we use the CADP toolset to check observation equivalence of models.

CADP Garavel et al. (2011) is a comprehensive toolbox for verifying models of concurrent systems, i.e., models consisting of multiple concurrent processes that together describe the overall system behavior. Relying on action-based semantic models, it offers functionalities covering the entire design cycle of concurrent systems: specification, simulation, rapid prototyping, verification, testing, and performance evaluation. It includes a wide range of verification techniques such as reachability analysis and compositional verification. CADP is used in this paper to check strong bisimulation equivalence of labeled transition systems. Two behavior deterministic Mealy machines are observation equivalent iff their associated labeled transition systems are strong bisimulation equivalent.

\subsection{Conformance learning with a conformance oracle}

The model-based testing (oracle) part of automata learning can be time consuming in practice. We therefore experimented with an alternative approach in which the model $M_{R}$ of the reference implementation $R$ is used as an oracle when learning a model for an implementation $I$. We will see that this use of what we call a conformance oracle may significantly speed up the learning process. 


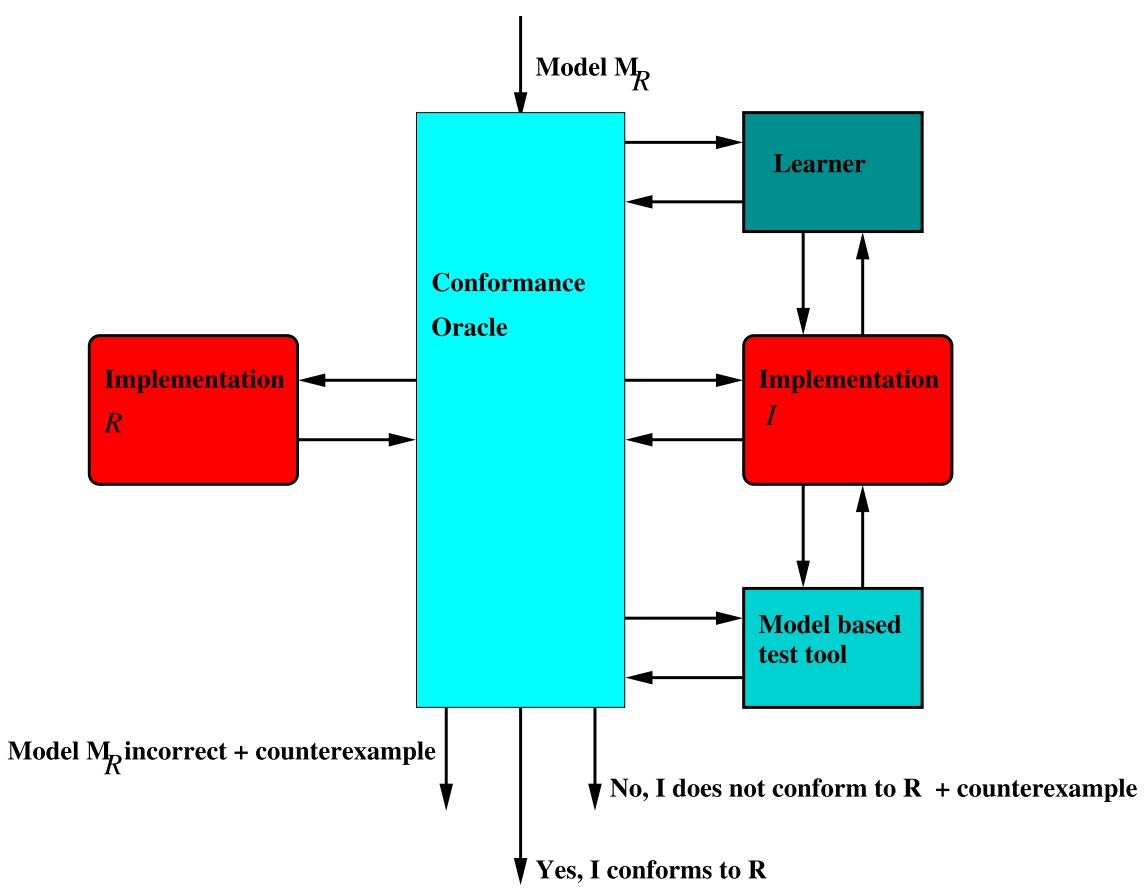

Fig. 5 Conformance oracle. The oracle takes a previously learned model $M_{R}$ for the reference $R$ as input and uses it to quickly answer equivalence queries by testing the equivalence of $M_{R}$ with the hypothesized model $M_{I}$ for the implementation $I$. When they are found to be inequivalent, the discovered counterexample is provided as input to $I$ and $R$ in order to test whether it proves the inequivalence of $R$ and $I, M_{I}$ and $I$, or $M_{R}$ and $R$

Suppose a learner has constructed an hypothesized Mealy machine model $M_{I}$ for implementation $I$. We want to use the availability of $M_{R}$ to speed up the validation (or counterexample discovery) for $M_{I}$, and reduce the use of the model-based test oracle as much as possible. Our approach works as follows:

1. We first use an equivalence checker to test $M_{R} \approx M_{I}$. If so, then we use a model-based test tool to further increase our confidence that $M_{I}$ is a good model of $I$. If modelbased testing reveals no counterexamples we are done, otherwise the learner may use a produced counterexample to construct a new model of $I$, and we return to step (1).

2. If $M_{R} \not \approx M_{I}$ then the equivalence checker produces an input sequence $u$ such that $o u t_{M_{R}}(u) \neq o u t_{M_{I}}(u)$. We apply $u$ to both implementations $R$ and $I$, and write $\operatorname{out}_{R}(u)$ and $\operatorname{out}_{I}(u)$, respectively, for the resulting output sequences.

3. If $\operatorname{out}_{R}(u) \neq \operatorname{out}_{M_{R}}(u)$ then model $M_{R}$ is incorrect and we may use counterexample $u$ to construct a new model for $R$.

4. Otherwise, if out $_{I}(u) \neq$ out $_{M_{I}}(u)$ then model $M_{I}$ is incorrect. In this case the learner may use counterexample $u$ to construct a new model for $I$, and we return to step (1).

5. Otherwise, we have identified an observable difference between implementations $R$ and $I$, i.e., $I$ is not conforming to reference implementation $R$.

Figure 5 illustrates the architectural embedding of a conformance oracle. 


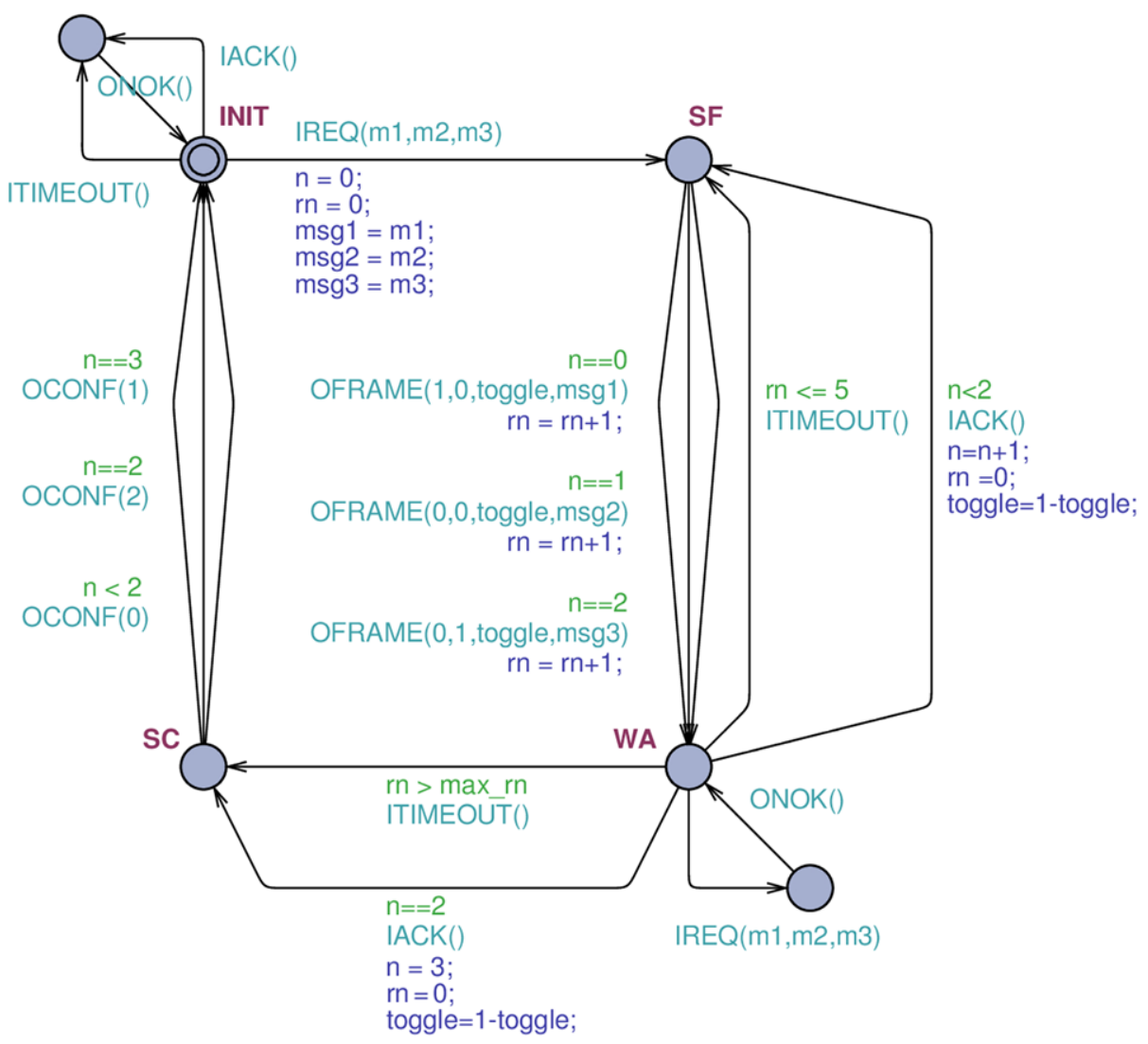

Fig. 6 Reference implementation of the BRP sender. The input symbols start with $I$, the output symbols start with $O$. In addition to symbols, the transitions contain value checks (or guards, $==,<,>$ ) and assignments $(=)$

\section{The BRP implementation and its mutants}

The bounded retransmission protocol (BRP) (Helmink et al. 1994; D'Argenio et al. 1997) is a variation of the well-known alternating bit protocol (Bartlett et al. 1969) that was developed by Philips Research to support infrared communication between a remote control and a television. In this section, we briefly recall the operation of the protocol, and describe the reference implementation of the sender and the 6 mutant implementations.

The bounded retransmission protocol is a data link protocol which uses a stop-and-wait approach known as 'positive acknowledgement with retransmission': after transmission of a frame the sender waits for an acknowledgement before sending a new frame. For each received frame the protocol generates an acknowledgement. If, after sending a frame, an acknowledgement fails to appear, the sender times out and retransmits the frame. An alternating bit is used to detect duplicate transmission of a frame.

Figure 6 illustrates the operation of our reference implementation of the sender of the BRP. Actually, the reference implementation that we used is a Java executable that was generated automatically from this diagram (represented as a Uppaal xml file, see Sect. 3). The sender protocol uses the following inputs and outputs: 
- Via an input IREQ $\left(m_{1}, m_{2}, m_{3}\right)$, the upper layer requests the sender to transmit a sequence $m_{1} m_{2} m_{3}$ of messages. For simplicity, our reference implementation only allows sequences of three messages, and the only messages allowed are 0 and 1 . When the sender is in its initial state INIT, an input IREQ $\left(m_{1}, m_{2}, m_{3}\right)$ triggers an output OFRAME $\left(b_{1}, b_{2}, b_{3}, m\right)$, otherwise it triggers output ONOK.

- Via an output OFRAME $\left(b_{1}, b_{2}, b_{3}, m\right)$, the sender may transmit a message to the receiver. Here $m$ is the actual transmitted message, $b_{1}$ is a bit that is 1 iff $m$ is the first message in the sequence, $b_{2}$ is a bit that is 1 iff $m$ is the last message in the sequence, and $b_{3}$ is the alternating bit used to distinguish new frames from retransmissions.

- Via input IACK the receiver acknowledges receipt of a frame and via input ITIMEOUT the sender is informed that a timeout has occurred, due to the loss of either a frame or an acknowledgement message. When the sender is in state WA ("wait for acknowledgement"), an input IACK or ITIMEOUT triggers either an output OFRAME $\left(b_{1}, b_{2}, b_{3}, m\right)$ or an output $\operatorname{OCONF}(i)$. If the sender is not in state WA, ONOK is triggered.

- Via an output OCONF $(i)$, the sender informs the upper layer about the way in which a request was handled:

$-i=0$ : the request has not been dispatched completely,

$-i=1$ : the request has been dispatched successfully,

$-i=2$ : the request may or may not have been handled completely; this situation occurs when the last frame is sent but not acknowledged.

An output OCONF occurs when either all three messages have been transmitted successfully, or when a timeout occurs after the maximal number of retransmissions.

Note that, within the state machine of Fig. 6, inputs and outputs strictly alternate. Thus it behaves like a Mealy machine. The state machine maintains variables msg1, msg2 and msg3 to record the three messages in the sequence, a Boolean variable toggle to record the alternating bit, an integer variable $n$ to record the number of messages that have been acknowledged, and an integer variable $r n$ to record the number of times a message has been retransmitted. Each message is retransmitted at most 5 times.

We consider the following six mutants of the reference implementation of the sender (see Appendix A):

1. Whereas the reference implementation only accepts a new request in the INIT state, mutant 1 also accepts new requests in state WA. Whenever mutant 1 receives a new request, the previous request is discarded and the sender starts handling the new one.

2. Whereas in the reference implementation each message is retransmitted at most 5 times, mutant 2 retransmits at most 4 times.

3. Whereas in the reference implementation the alternating bit is only toggled upon receipt of an acknowledgement, mutant 3 also toggles the alternating bit when a timeout occurs.

4. In mutant 4 the first and last control bit for the last message are swapped.

5. Mutant 5 outputs an $\operatorname{OCONF}(0)$ in situations where the reference implementation outputs OCONF(2).

6. If the first and the second message are equal then mutant 6 does not transmit the third message, but instead retransmits the first message.

Since input and output messages still alternate, all of the mutants still behave as Mealy machines. For all BRP implementations, we consider the inputs: IREQ $\left(m_{1}, m_{2}, m_{3}\right)$, IACK, and ITIMEOUT, where $m_{1}, m_{2}$, and $m_{3}$ can be either 0 or 1 . Thus, the input alphabet consists of 10 input symbols: 8 different IREQ inputs, one IACK input, and one ITIMEOUT input. We also have the following outputs: ONOK, OFRAME $\left(b_{1}, b_{2}, b_{3}, m\right)$, and $\operatorname{OCONF}(i)$, where $0 \leq i \leq 2$, i.e., 20 output symbols. In the next section, we discuss how to connect these implementations to an active Mealy machine learner and a model-based testing tool. 


\section{Experiments}

In this section, we report on the experiments that we did using LearnLib and JTorX to establish conformance of the six mutant implementations to the reference implementation.

Learning BRP models In order to learn models of the reference implementation and its mutants, we connect the implementations, which serve as SUT, to the LearnLib tool. ${ }^{1}$ Since all BRP implementations behave as Mealy machines, this is the type of state machine that we infer with our approach. In our experiments we consider the inputs: IREQ $\left(m_{1}, m_{2}, m_{3}\right)$, IACK, and ITIMEOUT, where $m_{1}, m_{2}$ and $m_{3}$ can be either 0 or 1 . Thus, the input alphabet consists of 10 input symbols: 8 different IREQ inputs, one IACK input, and one ITIMEOUT input. Moreover, we have the following outputs: ONOK, OFRAME $\left(b_{1}, b_{2}, b_{3}, m\right)$, where $b_{1}$, $b_{2}, b_{3}$ and $m$ can be either 0 or 1 , and $\operatorname{OCONF}(i)$, where $0 \leq i \leq 2$. In order to approximate equivalence queries, LearnLib provides several algorithms. We used the LearnLib test suite with randomly generated test traces containing 100 inputs.

The results of the inference of the reference implementation and the six mutants are shown in Table 2. For every implementation, we list the number of states in the learned model, as well as the average total number of Membership Queries (MQ). Moreover, we list the average total number of Test Traces (TT) generated for approximating equivalence queries. Note that these numbers do not include the last equivalence query, in which no counterexample has been found. Using CADP, we verified that all the learned models indeed are correct, i.e., equivalent to the Uppaal models described in Sect. 5. Each experiment was repeated 10 times with different seeds for the equivalence queries. For each measured value its average over the 10 experiments is listed in the table together with the standard deviation. If an experiment did not succeed to learn the model within two hours we aborted the experiment. In the last row we display how many of the 10 experiments did succeed. Even if an experiment did fail, we still use its numbers in calculating the average and the standard deviation, giving a lower bound for the real average and standard deviation for this experiment.

If we take a closer look at Table 2, we observe some interesting peculiarities. First, the number of test traces for mutant 1 is much higher than for the other implementations. The reason for this is that mutant 1 also accepts new requests in state WA. Whenever mutant 1 receives a new request, the previous request is discarded and the sender starts handling the new request. This makes it much harder to find a counterexample that produces an OCONF(0) or OCONF(2) output, since this requires six successive ITIMEOUT inputs without intermediate IREQ inputs. The probability that LearnLib selects (uniformly at random) six successive

\footnotetext{
${ }^{1}$ In previous work (Aarts et al. 2012b) we used TCP/IP socket communication. TCP/IP uses optimizations, TCP delayed acknowledgment technique and Nagle's algorithm, for reducing packet overhead. These optimizations slow down the communication in a setting with very small messages. In normal communication between SUT and learner the communication is an alternating pattern of sending input followed by a returned output. However in between two queries to the SUT an extra RESET input is sent, which breaks this alternating pattern. Exactly at that point the optimizations in TCP/IP cause a delay in communication to happen. Effectively this means that each query to the SUT gets an extra delay. By disabling the Nagle optimalization in the TCP/IP socket communication we can prevent these delays to happen. For even better performance we used direct method calls to the SUT by linking the SUT code against our learner. Removing this delay made the queries in the experiments much faster and therefore it was possible to learn the BRP models for larger retransmission counter and variable ranges in the same amount of time than in Aarts et al. (2012b). Moreover because membership queries in general are much shorter than equivalence testing queries, the performance gain per query in the first are much bigger than in the latter.
} 
Table 2 Learning statistics for the BRP reference implementation and mutants 1-6. MQ refers to the average total number of Membership Queries, TT to the average total number of Test Traces. vrX-Y means $m_{i} \in$ $[X \ldots Y]$, where $m_{i}$ is a message in $\operatorname{IREQ}\left(m_{1}, m_{2}, m_{3}\right)$, e.g. vr0-1 allows values 0 and 1 in a message in IREQ

\begin{tabular}{rlrrrrrrr}
\hline & RefImpl & Mut1 & Mut2 & Mut3 & Mut4 & Mut5 & Mut6 \\
\hline \multirow{2}{*}{ vr0-1 } & states & 156 & 156 & 128 & 156 & 156 & 156 & 136 \\
& avg. MQ & 24998 & 22174 & 19618 & 22488 & 24684 & 24998 & 21662 \\
& std.dev. & 1717 & 627 & 1006 & 769 & 1828 & 1717 & 736 \\
& avg. TT & 14 & 5830 & 7 & 15 & 14 & 14 & 14 \\
& std.dev. & 13 & 5142 & 6 & 13 & 13 & 13 & 13 \\
& Succeeded & $10 / 10$ & $10 / 10$ & $10 / 10$ & $10 / 10$ & $10 / 10$ & $10 / 10$ & $10 / 10$ \\
\hline
\end{tabular}

ITIMEOUT inputs in a row is low, since each time ITIMEOUT only has a $10 \%$ chance of being selected. This issue will be analyzed in more detail in Sect. 7. Second, the numbers for mutant 2 are slightly smaller than for the other implementations. The reason for this is that in mutant 2 the maximal number of retransmissions is smaller: 4 instead of 5, see Figs. 712. The size of the model and the times required for constructing and testing hypotheses (explored in the next section) all depend on the maximal number of retransmissions. This will be explored further in the next section.

More learning experiments Besides the maximal value of the retransmission counter, also changes in the domain of message parameters $m_{1}, m_{2}$, and $m_{3}$ will influence the learning results for the different implementations, because more inputs need to be considered. Therefore, we ran some additional experiments for different parameter settings of the reference implementation and mutant 1 (the behavior of mutants 2-6 is similar to that of the reference implementation, because no changes to the general structure of handling requests have been made). We evaluated how LearnLib performs for different maximal values for the retransmission counter rn. Moreover, we investigated what happens when increase the value range vr for each message parameter.

Table 3 and 4 show the results of learning models of the reference implementation and mutant 1 using different maximal numbers of retransmission and different value ranges for the message parameters $m_{1}, m_{2}$, and $m_{3}$. As expected, increasing the number of retransmissions rn and the value ranges vr both results in bigger models for which more membership and test traces are needed and, accordingly, more time for learning and testing. Increasing the value range leads to a fast growth in membership queries required to construct a hypothesis and the number of test traces required to find counterexamples for incorrect hypotheses, because the model to learn contains more states and the probability to select an ITIMEOUT or IACK input (which is needed in a counterexample) shrinks. For example, compare the results for rn15 and vr0-1 versus rn15 and vr0-2 in Table 3. Increasing the maximal number of retransmissions leads to a linear growth of membership queries required to construct a hypothesis, but to a fast growth of test traces required to find counterexamples for incorrect hypotheses, because the higher the retransmission counter, the more ITIMEOUT inputs are needed in a counterexample. For example, compare the results for $\mathrm{rn} 15$ to $\mathrm{rn} 20$ for $\mathrm{vr} 0-1$ in Table 3.

For mutant 1, the time needed for testing increases so fast that if the maximal number of retransmissions is 8 and there are 2 values, not every seed results in a correct model within 2 hours. In contrast, for 2 values the reference implementation only starts failing to learn 
Table 3 Learning statistics for reference implementation. MQ refers to the average total number of Membership Queries, TT to the average total number of Test Traces. vrX-Y means $m_{i} \in[X \ldots Y]$, where $m_{i}$ is a message in IREQ $\left(m_{1}, m_{2}, m_{3}\right)$, e.g. vr0-3 allows values $0,1,2$, and 3 in a message in IREQ. Similar, rn refers to the value of the retransmission counter in the model. Changing the number of retransmissions is done by increasing the value in the guard statement $(\mathrm{rn}>5$ and $\mathrm{rn} \leq 5)$ for the ITIMEOUT input from state WA to SF and from WA to SC

\begin{tabular}{|c|c|c|c|c|c|c|c|}
\hline & & rn15 & rn16 & rn17 & rn18 & rn19 & rn20 \\
\hline \multirow[t]{7}{*}{ vr0-1 } & states & 436 & 464 & 492 & 520 & 548 & 576 \\
\hline & avg. MQ & 83346 & 91949 & 99465 & 107206 & 108582 & 104113 \\
\hline & std.dev. & 4122 & 5807 & 4298 & 13624 & 6402 & 33896 \\
\hline & avg. TT & 20699 & 82854 & 103041 & 137750 & 295292 & 2665735 \\
\hline & std.dev. & 21865 & 129195 & 130379 & 180024 & 284401 & 3044574 \\
\hline & Success & $10 / 10$ & $10 / 10$ & $10 / 10$ & $10 / 10$ & $10 / 10$ & $9 / 10$ \\
\hline & & rn09 & rn10 & rn12 & rn13 & rn14 & rn15 \\
\hline \multirow[t]{7}{*}{ vr0-2 } & states & 730 & 808 & 964 & 1042 & 1120 & 1198 \\
\hline & avg. MQ & 755837 & 853000 & 1037250 & 1124192 & 1211588 & 564335 \\
\hline & std.dev. & 13562 & 18753 & 31762 & 37755 & 29241 & 603545 \\
\hline & avg. TT & 7507 & 20944 & 348407 & 1349612 & 2395184 & 7903428 \\
\hline & std.dev. & 5219 & 14340 & 460829 & 1882704 & 2608484 & 2833324 \\
\hline & Succeeded & $10 / 10$ & $10 / 10$ & $10 / 10$ & $10 / 10$ & $10 / 10$ & $4 / 10$ \\
\hline & & rn06 & rn07 & rn08 & rn09 & rn10 & rn11 \\
\hline \multirow[t]{7}{*}{ vr0-3 } & states & 1052 & 1220 & 1388 & 1556 & 1724 & 1892 \\
\hline & avg. MQ & 4971425 & 5789485 & 6641683 & 7445564 & 5975070 & 2433575 \\
\hline & std.dev. & 34020 & 84075 & 84468 & 105242 & 3413383 & 3341094 \\
\hline & avg. TT & 2587 & 14928 & 111122 & 502686 & 3795812 & 8129711 \\
\hline & std.dev. & 1682 & 9797 & 116094 & 545382 & 3528212 & 3376705 \\
\hline & Succeeded & $10 / 10$ & $10 / 10$ & $10 / 10$ & $10 / 10$ & $7 / 10$ & $2 / 10$ \\
\hline & & rn03 & rn04 & rn05 & rn06 & rn07 & rn08 \\
\hline \multirow[t]{7}{*}{ vr0-4 } & states & 994 & 1304 & 1614 & 1924 & 2234 & 2544 \\
\hline & avg. MQ & 16537318 & 21810721 & 27159739 & 32278521 & 37677908 & 27794859 \\
\hline & std.dev. & 56460 & 106048 & 102494 & 171051 & 277996 & 18521581 \\
\hline & avg. TT & 230 & 1392 & 13057 & 148571 & 1375285 & 4732741 \\
\hline & std.dev. & 246 & 1089 & 13744 & 162899 & 1056963 & 3445873 \\
\hline & Succeeded & $10 / 10$ & $10 / 10$ & $10 / 10$ & $10 / 10$ & $10 / 10$ & $6 / 10$ \\
\hline & & rn02 & rn03 & rn04 & rn05 & rn06 & \\
\hline \multirow[t]{7}{*}{ vr0-5 } & states & 1120 & 1636 & 2152 & 2668 & 3184 & \\
\hline & avg. MQ & 53959581 & 79140421 & 104476825 & 129818719 & 130303196 & \\
\hline & std.dev. & 0 & 106997 & 214990 & 232653 & 43041807 & \\
\hline & avg. TT & 106 & 1353 & 16010 & 98888 & 2591811 & \\
\hline & std.dev. & 77 & 1287 & 11680 & 116544 & 1743665 & \\
\hline & Succeeded & $10 / 10$ & $10 / 10$ & $10 / 10$ & $10 / 10$ & $6 / 10$ & \\
\hline & & rn02 & rn03 & rn04 & & & \\
\hline \multirow[t]{6}{*}{ vr0-6 } & states & 1712 & 2510 & 3308 & & & \\
\hline & avg. MQ & 205602133 & 302303504 & 353391698 & & & \\
\hline & std.dev. & 177195 & 259788 & 51494449 & & & \\
\hline & avg. TT & 294 & 6044 & 105437 & & & \\
\hline & std.dev. & 378 & 5491 & 65300 & & & \\
\hline & Succeeded & $10 / 10$ & $10 / 10$ & $5 / 10$ & & & \\
\hline
\end{tabular}


Table 4 Learning statistics for mutant 1. MQ refers to the average total number of Membership Queries, TT to the average total number of Test Traces. vrX-Y means $m_{i} \in[X \ldots Y]$, where $m_{i}$ is a message in $\operatorname{IREQ}\left(m_{1}, m_{2}, m_{3}\right)$, e.g. vr0-3 allows values $0,1,2$, and 3 in a message in IREQ. Similar, rn refers to the value of the retransmission counter in the model. Changing the number of retransmissions is done by increasing the value in the guard statement $(\mathrm{rn}>5$ and $\mathrm{rn} \leq 5)$ for the ITIMEOUT input from state WA to SF and from WA to SC

\begin{tabular}{|c|c|c|c|c|c|c|c|}
\hline & & rn03 & rn04 & rn05 & rn06 & rn07 & rn08 \\
\hline \multirow[t]{7}{*}{ vr0-1 } & states & 100 & 128 & 156 & 184 & 212 & 240 \\
\hline & avg. MQ & 13214 & 18199 & 22174 & 27999 & 32256 & 27365 \\
\hline & std.dev. & 402 & 515 & 627 & 738 & 850 & 16821 \\
\hline & avg. TT & 154 & 1250 & 5830 & 61495 & 667761 & 7903190 \\
\hline & std.dev. & 129 & 1615 & 5142 & 78465 & 516786 & 3371144 \\
\hline & Succeeded & $10 / 10$ & $10 / 10$ & $10 / 10$ & $10 / 10$ & $10 / 10$ & $6 / 10$ \\
\hline & & rn02 & rn03 & rn04 & rn05 & & \\
\hline \multirow[t]{7}{*}{ vr0-2 } & states & 184 & 262 & 340 & 418 & & \\
\hline & avg. MQ & 171318 & 243928 & 326408 & 334443 & & \\
\hline & std.dev. & 1603 & 2281 & 2960 & 131246 & & \\
\hline & avg. TT & 347 & 9655 & 278077 & 4388168 & & \\
\hline & std.dev. & 331 & 8693 & 159918 & 2974071 & & \\
\hline & Succeeded & $10 / 10$ & $10 / 10$ & $10 / 10$ & $8 / 10$ & & \\
\hline & & rn02 & rn03 & rn04 & & & \\
\hline \multirow[t]{6}{*}{ vr0-3 } & states & 380 & 548 & 716 & & & \\
\hline & avg. MQ & 1730589 & 2495661 & 1272022 & & & \\
\hline & std.dev. & 0 & 0 & 1017988 & & & \\
\hline & avg. TT & 3312 & 224096 & 6522672 & & & \\
\hline & std.dev. & 3031 & 246072 & 2488837 & & & \\
\hline & Succeeded & $10 / 10$ & $10 / 10$ & $2 / 10$ & & & \\
\hline
\end{tabular}

the correct model within hours for all seeds for the maximal number of retransmissions of 20. As mentioned before, the reason for this is that mutant 1 also accepts new requests in state WA and discards previous requests. Also in the case where the maximal number of retransmissions is 5 and there are 3 values, LearnLib is not able to construct a correct model for all seeds for mutant 1 within 2 hours. This is not surprising, because in both cases the probability to select a counterexample is even lower than for mutant 1 in Table 2. Once LearnLib fails to learn a correct model, we assume that it will also fail for larger value ranges of the parameters. The same holds for the retransmission counter. Once some of the experiments fail, the number of successful runs with a higher retransmission counter will decrease.

Conformance checking We compare the two methods, described in Sect. 4.1, for establishing the conformance of the mutant implementations to the reference implementation of BRP. We only consider the versions of the models with at most 5 retransmissions and 2 different parameter values. 
Table 5 Counterexamples found by equivalence checking of mutant models and reference implementation $(\mathrm{IT}=\mathrm{ITIMEOUT}, \mathrm{IR}=\mathrm{IREQ}, \mathrm{IA}=\mathrm{IACK}, \mathrm{OF}=$ OFRAME $)$

\begin{tabular}{|c|c|c|c|}
\hline & counterexample & output & expected \\
\hline Mut1 & $\operatorname{IR}(0,0,0) \operatorname{IR}(0,0,0)$ & $\mathrm{OF}(1,0,0,0)$ & ONOK() \\
\hline Mut2 & $\operatorname{IR}(0,0,0) \operatorname{IT}() \operatorname{IT}() \operatorname{IT}() \operatorname{IT}() \operatorname{IT}()$ & OCONF(0) & $\mathrm{OF}(1,0,0,0)$ \\
\hline Mut3 & $\operatorname{IR}(0,0,0) \operatorname{IT}()$ & $\mathrm{OF}(1,0,1,0)$ & $\mathrm{OF}(1,0,0,0)$ \\
\hline Mut4 & $\operatorname{IR}(0,0,0) \operatorname{IA}() \operatorname{IA}()$ & $\mathrm{OF}(1,0,0,0)$ & $\mathrm{OF}(0,1,0,0)$ \\
\hline Mut5 & $\operatorname{IR}(0,0,0) \operatorname{IA}() \operatorname{IA}() \operatorname{IT}() \operatorname{IT}() \operatorname{IT}() \operatorname{IT}() \operatorname{IT}() \operatorname{IT}()$ & OCONF $(0)$ & OCONF(2) \\
\hline Mut6 & $\operatorname{IR}(0,0,1) \operatorname{IA}() \operatorname{IA}()$ & $\mathrm{OF}(0,1,0,0)$ & $\mathrm{OF}(0,1,0,1)$ \\
\hline
\end{tabular}

Table 6 Conformance testing the mutants with JTorX for retransmission counter 5 and value range 0-1: using the reference model JTorX will generate a test sequence and applies it to the mutant implementation in order to establish the conformance between the reference model and the mutant. For all mutants it detected the non-conformance and returned a counterexample. The table shows the average number of IO symbols, i.e. the average number of inputs and outputs, needed to find the counterexample for each mutant

\begin{tabular}{llllllll}
\hline & & mut1 & mut2 & mut3 & mut4 & mut5 & mut6 \\
\hline vr0-1 & avg. IO symbols & 5 & 894 & 19 & 37 & 6657 & 198 \\
rn05 & std.dev. & 1 & 768 & 12 & 20 & 4983 & 268 \\
\hline
\end{tabular}

The first method, conformance learning, used the CADP (bisimulation) equivalence checker to compare the model $M_{I}$, that we learned for the mutant implementations $I$, with the model $M_{R}$ learned for the reference implementation $R .^{2}$ For each of the mutants, CADP quickly found a counterexample trace illustrating the difference between the models of the mutant and the model of the reference implementation (for each mutant it takes around 3 seconds to find the counterexample). The counterexamples found by CADP are depicted in Table 5.

The second method, conformance model-based testing, used the model $M_{R}$ of the reference implementation $R$ as input for the JTorX model-based testing tool and the mutant implementations $I$ as SUTs. Test steps were executed until a counterexample was found. Again, JTorX found a counterexample for each of the mutant implementations. The average number of IO symbols, i.e. the average number of inputs and outputs in the counterexample, is shown in Table 6 for the different mutant implementations. Because JTorX generates a single long testing sequence without intermediate resets, the resulting counterexamples are rather long sequences and therefore are not shown in the table.

In a JTorX experiment the performance is measured in number of IO symbols whereas in a Learnlib experiment the performance is measured in number of membership and testing queries. Comparing the measurements of both experiments is not trivial. Although each testing query corresponds to $200 \mathrm{IO}$ symbols, since we use a random test suite generating test traces with 100 input symbols, each membership query varies in length determined by the learning algorithm. However, in a crude approximation we could say that in the learning experiment \#IO symbols $\approx x * M Q+200 * T T$ where $x$ is a guessed factor of the average length of the membership queries.

\footnotetext{
${ }^{2}$ Essentially the same counterexamples were also found using the JTorX iocoChecker. The JTorX iocoChecker, which is distributed as an extra feature of the JTorX model-based testing tool (Belinfante 2010), is a tool to check analytically whether two models are ioco-related.
} 
Table 7 Conformance checking using the equivalence checker CADP for retransmission counter 10 and value range 0-1. MQ refers to the average total number of Membership Queries, TT to the average total number of Test Traces. The table shows the learning statistics for reference implementation and mutants 3-6. After learning the reference and mutant models we could find in around 3 seconds a counterexample trace illustrating the difference between the model of the mutant and the model of the reference implementation

\begin{tabular}{llrrrrr}
\hline & RefImpl & Mut3 & Mut4 & Mut5 & Mut6 \\
\hline vr0-1 & states & 296 & 296 & 296 & 296 & 256 \\
rn10 & avg. MQ & 55417 & 46509 & 55417 & 55417 & 47166 \\
& std.dev. & 3767 & 1899 & 3767 & 3767 & 3083 \\
& avg. TT & 280 & 357 & 280 & 280 & 280 \\
& std.dev. & 171 & 266 & 171 & 171 & 171 \\
& Succeeded & $10 / 10$ & $10 / 10$ & $10 / 10$ & $10 / 10$ & $10 / 10$ \\
\hline
\end{tabular}

Table 8 Conformance testing the mutants with JTorX for retransmission counter 10 and value range 0-1: using the reference model JTorX will generate a test sequence and applies it to the mutant implementation in order to establish the conformance between the reference model and the mutant. For all mutants it detected the non-conformance and returned a counterexample. The table shows the average number of IO symbols, i.e. the average number of inputs and outputs, needed to find the counterexample for each mutant

\begin{tabular}{lllllrr}
\hline & & mut1 & mut3 & mut4 & mut5 & mut6 \\
\hline vr0-1 & avg. IO symbols & 5 & 19 & 37 & 109154 & 178 \\
rn10 & std.dev. & 1 & 12 & 20 & 90617 & 228 \\
\hline
\end{tabular}

If we look at Table 6 we immediately see that the number of IO symbols needed for mutant 5 is much bigger than for the other mutants. However, Table 2 shows that learning mutant 5 is not more difficult than learning the other mutants. This can be explained by the fact that if we look at the counterexample for mutant 5 in Table 5 we immediately see that the counterexample for mutant 5 is the longest. When looking at the model for mutant 5 we can see that the number of ITIMEOUT inputs in the counterexample is directly related to the value of the retransmission counter $\mathbf{r n}$.

To investigate the effects, we increase the retransmission counter rn to 10 . The learning results for $r n=10$ are shown in Table 7 for the different mutants and for conformance testing the mutants with JTorX in Table 8. Note that in learning the mutants for the retransmission counter of 10 we skipped mutant 1 , because it was already shown in Table 2 that it couldn't be learned in 2 hours, and we skipped mutant 2 because the only difference between mutant 2 and the reference implementation is a different retransmission number.

After learning the new mutants CADP could again find in around 3 seconds a counterexample trace illustrating the difference between the models of the mutants and the model of the reference implementation. The results in Table 7 show that learning mutant 5 with a larger retransmission counter still is not more difficult than learning the other mutants, whereas Table 8 shows that for mutant 5 model-based testing still requires much longer traces than for the other mutants. However, when comparing Table 7 and Table 8 it shows that for all mutants the number of IO symbols is only increased for mutant 5 .

Again, this can be explained by the fact that if we look at the counterexample for mutant 5 in Table 5 we immediately see that the number of ITIMEOUT inputs in the counterexample is directly related to the value of the retransmission counter $\mathbf{r n}$. By increasing the retransmission counter rn, the counterexample for mutant 5 becomes longer, and since finding longer 
counterexamples takes longer, this increases the time required by JTorX to find a counterexample. In contrast, for mutants 1, 3, 4 and 6 the counterexamples have no ITIMEOUT inputs and are independent of the retransmission counter rn, which explains why in the experiments for these mutants the number of IO symbols for finding counterexamples doesn't increase for a larger retransmission counter $\mathbf{r n}$.

Thus by comparing Table 7 and Table 8 we see that the increase in the number of IO symbols needed for conformance testing of mutant 5 is big. However, this number of IO symbols needed for conformance testing of mutant 5 is comparable in size to the number of membership and testing queries for learning mutant 5: compare $109154 \mathrm{IO}$ symbols from JTorX to $111417=1 \cdot 55417+200 \cdot 280(\# I O$ symbols $\approx x \cdot M Q+200 \cdot T T)$ IO symbols from LearnLib, where we assume a lower bound $x=1$ on the average length of membership queries.

Since LearnLib tests hypotheses in a way similar to JTorX and because the counterexample of mutant 5 reaches the deepest part of the model, testing during learning the model with LearnLib of mutant 5 and conformance testing with JTorX of mutant 5 become even difficult. Therefore their performance in both experiments becomes comparable.

In general, however, learning models of proposed implementations takes more time than model-based testing them but also provides more information in the form of a learned model. Even in these cases, it may still be beneficial to use learning tools since the learned models can for instance be used for model checking analysis.

\section{Further analysis and improvements}

\subsection{Why random testing sometimes fails}

In our experiments, the most effective technique available in LearnLib for approximating equivalence queries turned out to be random testing. In order to analyze the effectiveness of this method, we may compute the probabilities of reaching states that provide a counterexample within a certain number of transitions, by translating the Mealy machine of the teacher (the system under test) into a discrete time Markov chain (DTMC). This DTMC has the same states as the Mealy machine, and the probability of going from state $q$ to state $q^{\prime}$ is equal to the number of transitions from $q$ to $q^{\prime}$ in the Mealy machine divided by the total number of inputs. Through analysis of this DTMC, the MRMC model checker can compute the probability of finding certain counterexamples within a given time.

MRMC (Katoen et al. 2011) is a probabilistic model checker, which can be used to check the probability that a logical statement (such as a system breakdown) occurs in a given continuous- or discrete-time Markov chain, with or without reward functions (common in Markov decision processes (Puterman 1994)). Such a logical statement can be expressed in a probabilistic branching-time logic PCTL (Hansson and Jonsson 1994) or CSL (Aziz et al. 1996). The probabilistic models may also contain reward functions and bounds on these can be checked in combination with the time and probability values.

We use MRMC to compute the probability of reaching certain states in an implementation within a certain number of steps in a setting where inputs are generated randomly. We wrote a small script that converts LTSs in . aut format to DTMCs in . tra/ . lab format, which are accepted as input by MRMC.

Using MRMC we computed that for the reference implementation with up to 7 retransmissions the probability of reaching, within a single test run of 125 steps, a state with an 
Table 9 Equivalence query statistics for mutant 1 with TorXakis. vrX-Y means $m_{i} \in[X \ldots Y]$, where $m_{i}$ is a message in $\operatorname{IREQ}\left(m_{1}, m_{2}, m_{3}\right)$, e.g. vr0-2 allows values 0,1 , and 2 in a message in IREQ. Similar, rn refers to the value of the retransmission counter in the model. Changing the number of retransmissions is done by increasing the value in the guard statement $(\mathrm{rn}>5$ and $\mathrm{rn} \leq 5)$ for the ITIMEOUT input from state WA to SF and from WA to SC

\begin{tabular}{llll}
\hline & & rn5 & rn7 \\
\hline vr0-1 & (avg. \#test symbols, st. dev) & $(1785,1921)$ & $(19101,22558)$ \\
vr0-2 & (avg. \#test symbols, st. dev) & $(2991,2667)$ & $(18895,15158)$ \\
vr0-9 & (avg. \#test symbols, st. dev) & $(3028,3199)$ & \\
\hline
\end{tabular}

outgoing OCONF $(0)$ transition is 0.0247121 . This means the probability of reaching a state with an outgoing OCONF(0) transition within 75 test runs is 0.847 . This result explains why LearnLib requires very few test runs to learn a correct model of this system, which it does within seconds. Using MRMC, we also computed that for the version of mutant 1 with up to 7 retransmissions the probability of reaching, within 125 steps, a state with an outgoing OCONF(0) transition is only 0.0000010 . Hence, finding a counterexample by random testing will take much longer for mutant 1 than for the reference, explaining why LearnLib needs 667761 (see Table 4) test runs on average to find this counterexample $\left(0.999999^{667761} \approx 0.51\right.$ is the probability of not finding this trace in 667761 tries $)$.

\subsection{Using abstraction to speed up testing}

A simple way to increase the probability of finding counterexamples for mutant 1 within a short time is to increase the probability of ITIMEOUT inputs, for instance by assigning an equal probability of $1 / 3$ to the ITIMEOUT, IACK and IREQ inputs. Symbolic testing tools and abstraction learners will use this distribution, because they initially assume that the parameter values are not as important as the input types. TorXakis and Tomte (see Sect. 3) are such tools and we therefore evaluated the effect of using these instead of the basic random testing implemented in LearnLib.

TorXakis For the experiments above we employed LearnLib for both the learning and testing phase. However, it is also possible to perform the equivalence check by an external tool. We experimented with the LTS-based model-based testing tool TorXakis to see whether we can improve on detecting incorrect hypothesis models.

Table 9 summarizes the results obtained with TorXakis when testing mutant 1 against the hypothesized LearnLib model for $r n 5$, vr $0-1, r n 7, v r 0-1$ and $r n 5, v r 0-2$. In addition, the results for the scenarios $r n 5, v r 0-9$ and $r n 7, v r 0-2$ are presented. LearnLib did not manage to find a counterexample in these cases. The numbers in Table 9 are the average lengths of the test runs, measured in test symbols (both input and output), until a discrepancy between the model and mutant 1 was detected. The average is taken over 10 different random test runs. We do not measure the timing, because it makes no sense for TorXakis: TorXakis explicitly tests for non-occurrence of outputs by means of a time-out, and while the time value chosen for this time-out is in some sense arbitrary, it has a very strong influence on the total duration of a test.

TorXakis is able to detect counterexamples for the incorrect hypothesized models for $r n 5, v r 0-1, r n 7, v r 0-1$, and for $r n 5, v r 0-2$. Only after the IREQ input has been selected, the message values are randomly selected. This implies that increasing the domain of possible message values does not increase the length of the test case required to detect the 
Table 10 Learning statistics for mutant 1 using Tomte. vrX-Y means $m_{i} \in[X \ldots Y]$, where $m_{i}$ is a message in IREQ $\left(m_{1}, m_{2}, m_{3}\right)$, e.g. vr0-1 allows values 0 and 1 in a message in IREQ. rn refers to the value of the retransmission counter in the model. Changing the number of retransmissions is done by increasing the value in the guard statement $(\mathrm{rn}>5$ and $\mathrm{rn} \leq 5)$ for the ITIMEOUT input from state WA to SF and from WA to SC

\begin{tabular}{rlrrrrrr}
\hline & rn09 & rn10 & rn12 & rn13 & rn14 & rn15 \\
\hline vr0- $\infty$ & states & 62 & 68 & 80 & 86 & 92 & 98 \\
& avg. MQ & 1518 & 1875 & 2449 & 2629 & 2196 & 1377 \\
& std.dev. & 3 & 4 & 3 & 4 & 1372 & 1564 \\
& avg. TT & 1175 & 3525 & 51589 & 89133 & 182508 & 258552 \\
& std.dev. & 1998 & 2816 & 34687 & 33648 & 82534 & 78432 \\
& Succeeded & $10 / 10$ & $10 / 10$ & $10 / 10$ & $10 / 10$ & $7 / 10$ & $4 / 10$ \\
\hline
\end{tabular}

counterexample. Combined with the fact that TorXakis generates one very long test case, it is able to find a counterexample for the scenario $r n 7, v r 0-2$ within reasonable time. In conclusion, TorXakis is able to detect counterexamples within reasonable time, which LearnLib could not detect.

Tomte Through the use of counterexample abstraction refinement, Tomte is able to learn models for a restricted class of extended finite state machines in which one can test for equality of data parameters, but no operations on data are allowed. The current version of Tomte requires that only the first and the last occurrence of parameters of actions is remembered. As a result, Tomte can only learn models such as mutant 1, where each IREQ input overwrites previous occurrences of the message parameters. For these instances, however, Tomte outperforms LearnLib with several orders of magnitude. Table 10 gives an overview of the statistics for learning mutant 1 with Tomte. Since in Tomte the entire range of message values for mutant 1 is abstracted into a single equivalence class, Tomte needs far fewer queries than LearnLib (cf. Table 4) and, moreover, Tomte can handle a larger value range than LearnLib: compare the results vr0- $\infty$ used by Tomte to vr0-3 used by LearnLib. Work is underway to extend Tomte so that it can also learn the BRP reference implementation and the other mutants.

\subsection{Conformance learning with a conformance oracle}

Above, we demonstrated how to make random testing a little smarter by making use of abstractions. This results in a speed up in the time random testing requires to find a counterexample, but even this method has its limits as shown in Table 10. We now show that we can remove random testing altogether using the notion of a conformance oracle using the method 'Conformance learning with a conformance oracle' described in Sect. 4.2, in which we use CADP as an equivalence checker.

The results of our experiments can be found in Table 11 in which for each mutant we found non-conformance with the reference implementation. The number of states listed are the number of states of the last hypothesis found in step 5 of the conformance learning with a conformance oracle algorithm described in Sect. 4.2. Compared with Table 10, we only have the number of states and the number of membership queries listed, because when using a conformance oracle we do no random testing after learning anymore. Thus, by using a conformance oracle instead of random testing, the learning process becomes completely deterministic and, therefore, we only had to do each experiment in Table 11 once. 
Table 11 Finding counterexamples using a conformance oracle, case $\mathbf{r n}=5$. In fact we are learning the mutant using the reference model as a conformance oracle until we found a counterexample which proves both the learned mutant model and the mutant implementation different from the reference implementation

\begin{tabular}{|c|c|c|c|c|c|c|c|}
\hline & & mut1 & mut2 & mut3 & mut4 & mut5 & mut6 \\
\hline vr0-1 & states & 16 & 127 & 16 & 16 & 155 & 20 \\
\hline rn05 & MQ & 1771 & 17798 & 1771 & 1771 & 21718 & 2211 \\
\hline
\end{tabular}

Table 12 Finding a counterexample for mutant 5 using a conformance oracle. In fact we are learning the mutant using the reference model as a conformance oracle until we found a counterexample which proves both the learned mutant model and the mutant implementation different from the reference implementation

\begin{tabular}{|c|c|c|c|c|c|c|c|}
\hline & & rn09 & rn10 & rn11 & rn12 & rn13 & rn14 \\
\hline \multirow[t]{2}{*}{ vr0-1 } & states & 267 & 295 & 323 & 351 & 379 & 407 \\
\hline & MQ & 42751 & 50189 & 54949 & 63227 & 68267 & 77385 \\
\hline
\end{tabular}

Table 13 Conformance testing mutant 5 using JTorX. vrX-Y means $m_{i} \in[X \ldots Y]$, where $m_{i}$ is a message in IREQ $\left(m_{1}, m_{2}, m_{3}\right)$, e.g. vr0-1 allows values 0 and 1 in a message in IREQ. rn refers to the value of the retransmission counter in the model. Changing the number of retransmissions is done by increasing the value in the guard statement $(\mathrm{rn}>5$ and $\mathrm{rn} \leq 5)$ for the ITIMEOUT input from state WA to SF and from WA to SC

\begin{tabular}{|c|c|c|c|c|c|c|c|}
\hline & & rn09 & rn10 & rn11 & rn12 & rn13 & rn14 \\
\hline \multirow[t]{2}{*}{ vr0-1 } & avg. IO symbols & 54701 & 109154 & 141285 & 475398 & 497128 & 1066146 \\
\hline & std.dev. & 39385 & 90617 & 95454 & 475627 & 541333 & 803093 \\
\hline
\end{tabular}

Table 11 shows that finding a counterexample using the conformance oracle is the most difficult for mutants 2 and 5, however, it is nearly trivial for the other four mutants, because for these mutants the final hypothesis only has between 16-20 states. We further investigate mutant 5 by scaling up the rn parameter in order to discover the limits of using the conformance oracle setup and to compare its performance to that of a state-of-the-art model-based testing tool.

Table 5 shows that for mutant 5 the counterexample does depend on the rn parameter: with a higher rn value, more ITIMEOUT inputs are required in the counterexample for proving mutant 5 to be different from the reference implementation with the same rn value. We investigate mutant 5 by doing both conformance model-based testing and conformance learning using a conformance oracle for increasing rn values as shown in Tables 12 and 13.

Testing using a conformance oracle is really quick: the experiment typically runs within a few seconds. Furthermore, the number of membership queries required to build the hypothesis seems to increase only linearly with increasing rn. In contrast, as Table 13 shows, the number of IO symbols and its related testing time required by JTorX increases much faster. The reason for this difference between the two methods is that for conformance testing the time required depends on the probability of reaching a state that provides a counterexample, while learning using a conformance oracle is fully deterministic and thus is independent of this probability. 


\section{Conclusion}

We show how to apply active state machine learning methods to a real-world use case from software engineering: conformance testing implementations of the bounded retransmission protocol (BRP). To the best of our knowledge, this use of active learning methods is entirely novel. We demonstrate how to make this application work by combining active learning algorithms (LearnLib and Tomte) with tools from verification (an equivalence checker, CADP) and testing (a model-based test tool, JTorX).

A nice property of the BRP is that it contains two parameter values (the number of retransmissions $\mathbf{r n}$ and the range of message values vr), which can be increased to obtain increasingly complex protocols. This makes it an ideal use case for state machine learning methods because it allows us to discover the limits of their learning capabilities. We investigated these limits on testing the conformance of six mutant implementations with respect to a given reference implementation. All implementations were treated as black-box software systems. The goal of our experiments was to discover how active learning tools can be used to establish the conformance between the mutant and reference implementations. The results of these experiments can be summarized as follows:

- The problem of test selection is a big bottleneck for state-of-the-art active learning tools. Existing model-based testing tools can be used to make this bottleneck less severe.

- Increasing the number of message values vr (the alphabet size) increases the time required for test selection as well as the time needed for finding a hypothesis (using membership queries).

- Increasing the maximal number of retransmissions rn (the length of counterexamples) increases the time required for test selection much more than the time needed for finding a hypothesis.

- Establishing conformance using an equivalence checker and two learned models is very fast. Using only a single learned model and a model-based testing tool is also fast, but can run into problems because test selection takes much longer than equivalence checking.

- Interestingly, there are cases where learning a mutant model (and checking equivalence) is as fast as model-based conformance testing. In general, however, learning a single model and subsequent testing is faster than learning two models.

Furthermore, we noticed in our experiments that the state-of-the-art LearnLib active learning tool quickly runs into trouble when learning one of these mutant implementations. This case was analyzed separately using a probabilistic model checker (MRMC), and based on this analysis we suggested two ways of improving the performance of the active learning method: using a state-of-the-art model-based test tool (TorXakis) for symbolic evaluation of equivalence queries, using a new learning method based on abstraction refinement (Tomte), and introducing a new way of learning based on the novel concept of a conformance oracle. Such a conformance oracle effectively learns two models at once and uses the (partially) learned models in an equivalence checker to quickly answer equivalence queries asked by either learner. When the models are similar, this can greatly reduce the cost of learning.

The concept of a conformance oracle opens up several interesting directions for future work. In particular, since it can also be used as a model-based tester, it would be interesting to further investigate exactly when and why it can be used to establish conformance more quickly than state-of-the-art model-based test tools. Our study already found one such case in mutant 5, where tools based on random testing are troubled by the low probability of reaching a state that leads to a counterexample. A conformance oracle in combination with an active learning tool finds the same counterexample deterministically and in fewer steps. 
The concept is also closely linked to transfer learning: it can use a previously learned model to speed up the process of learning a new (similar) model. A conformance oracle, however, can transfer these models during the learning process itself, making it an interesting approach for distributed learning settings.

The BRP use case, including the models as well as all the scripts used to link the different tools together, have been made available on-line for testing and learning by fellow researchers at http://www.italia.cs.ru.nl.

Acknowledgements Supported by STW project 11763 Integrating Testing And Learning of Interface Automata (ITALIA).

We thank Colin de la Higuera and Bernard Steffen for suggesting to use the learned reference model for answering equivalence queries, and Axel Belinfante for assisting us with JTorX.

\section{Appendix A: Mutants of the BRP sender}

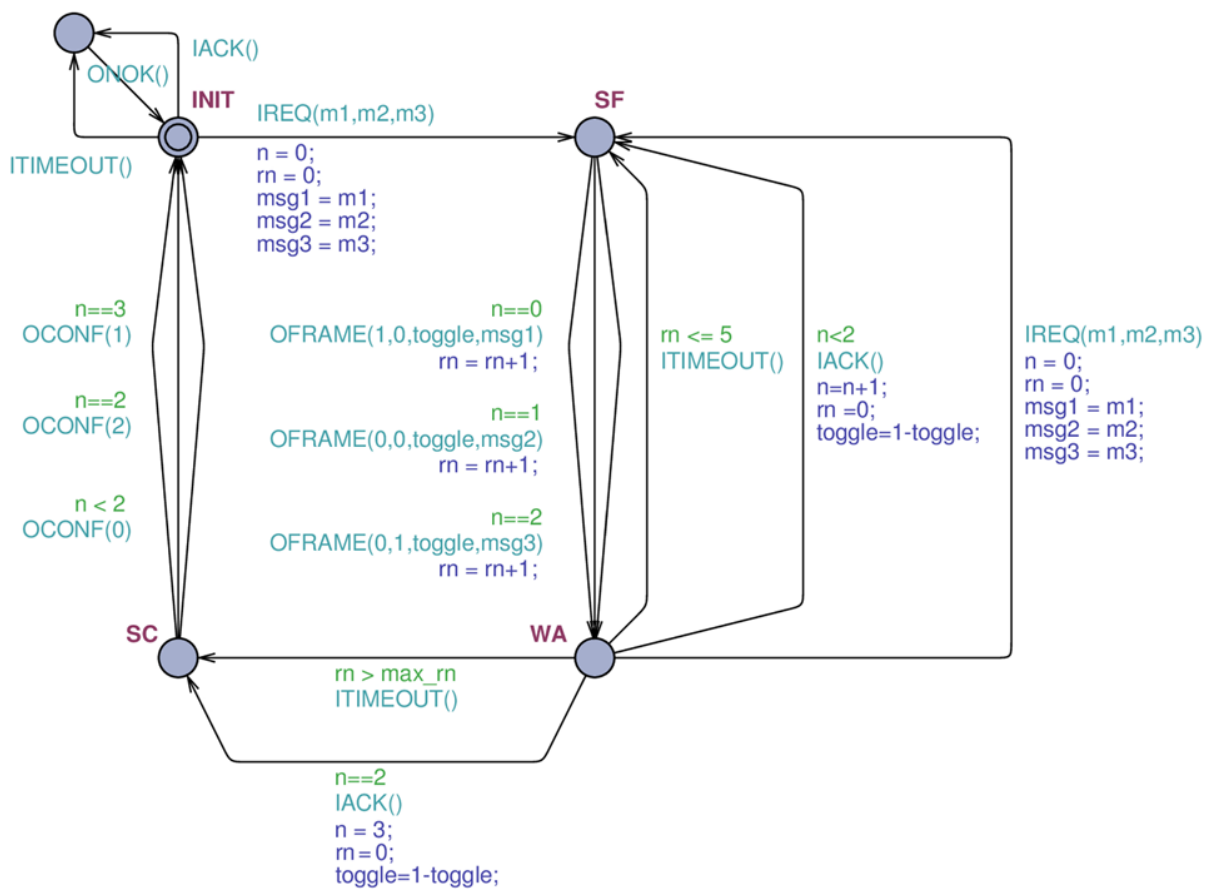

Fig. 7 Mutant 1 


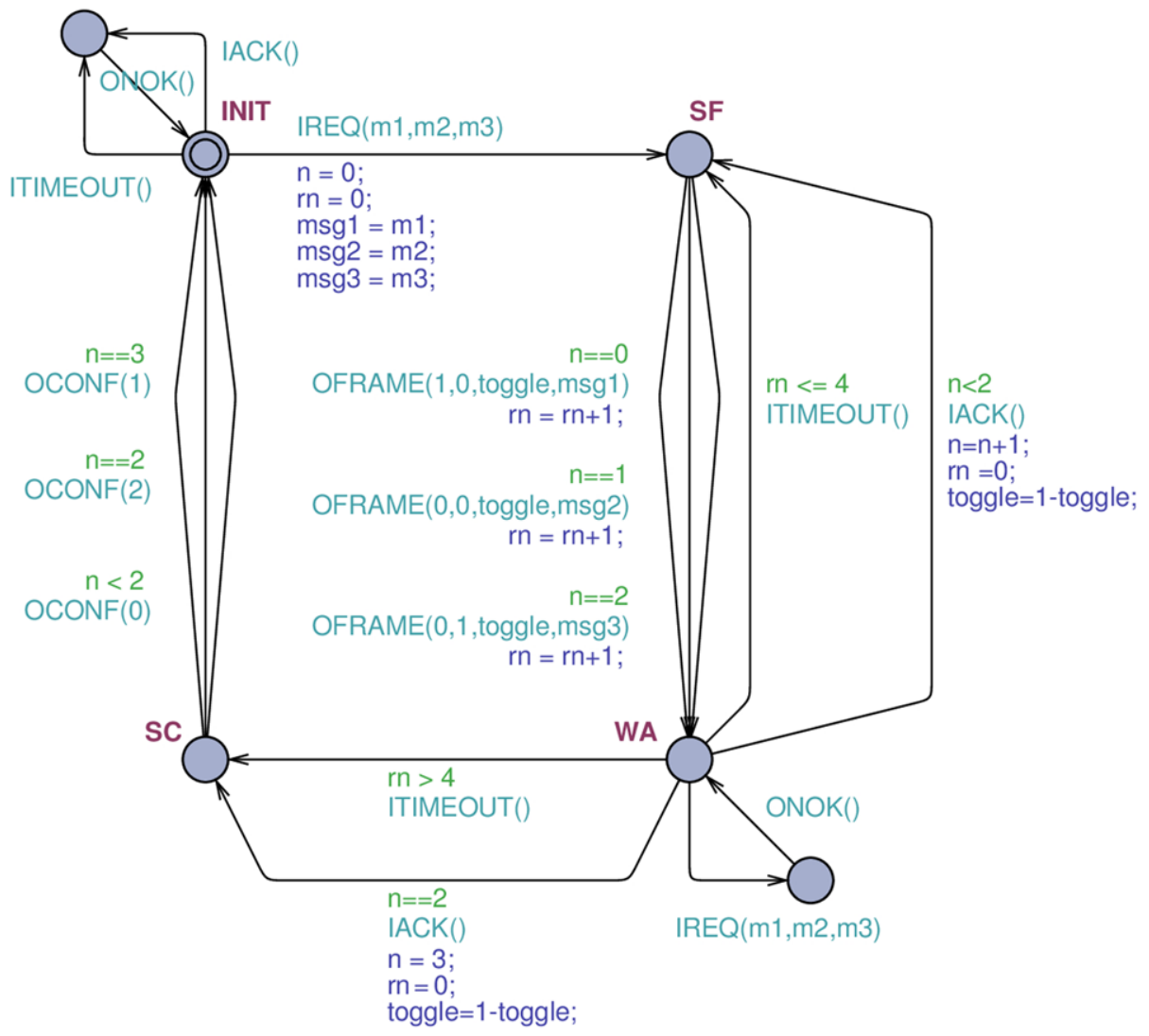

Fig. 8 Mutant 2 


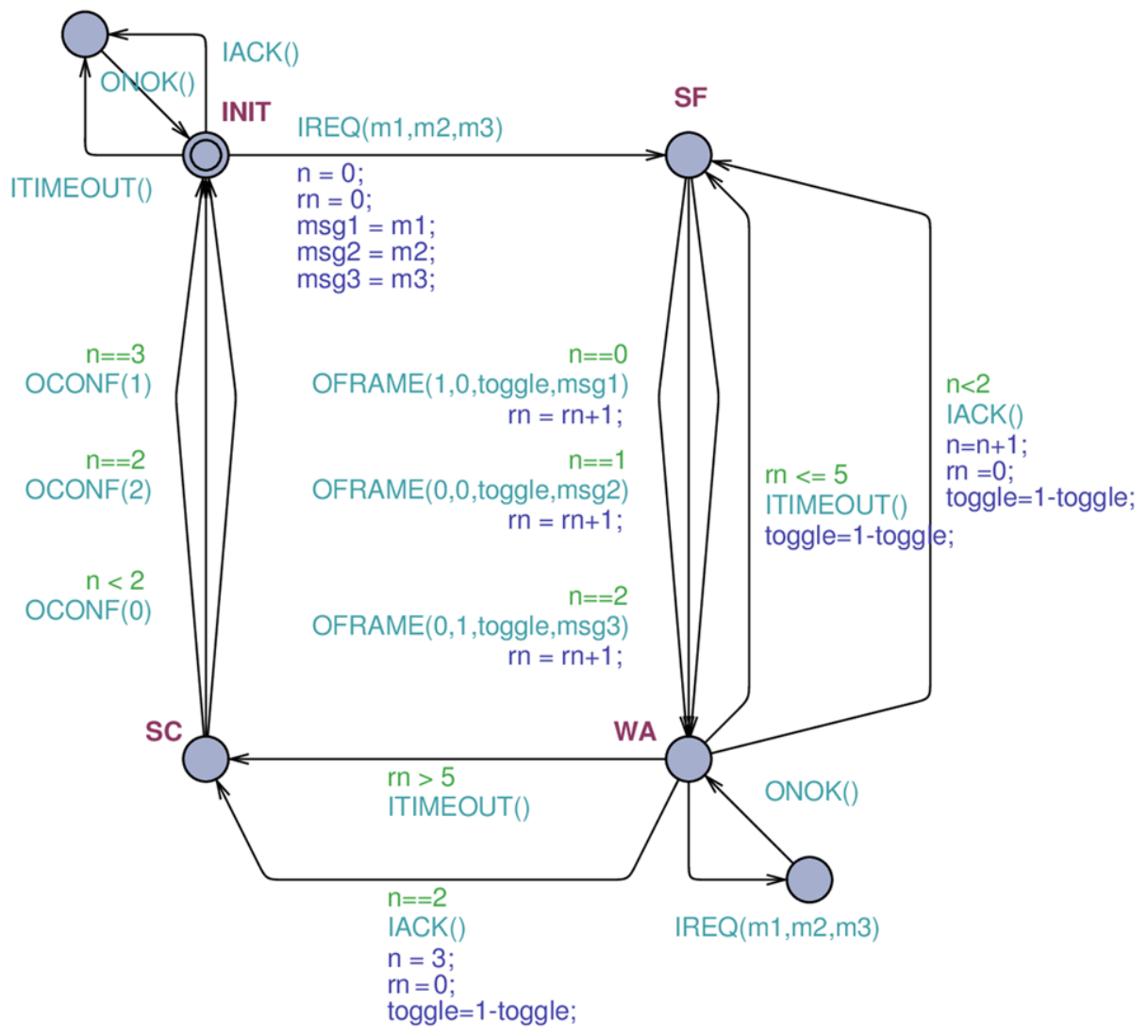

Fig. 9 Mutant 3 


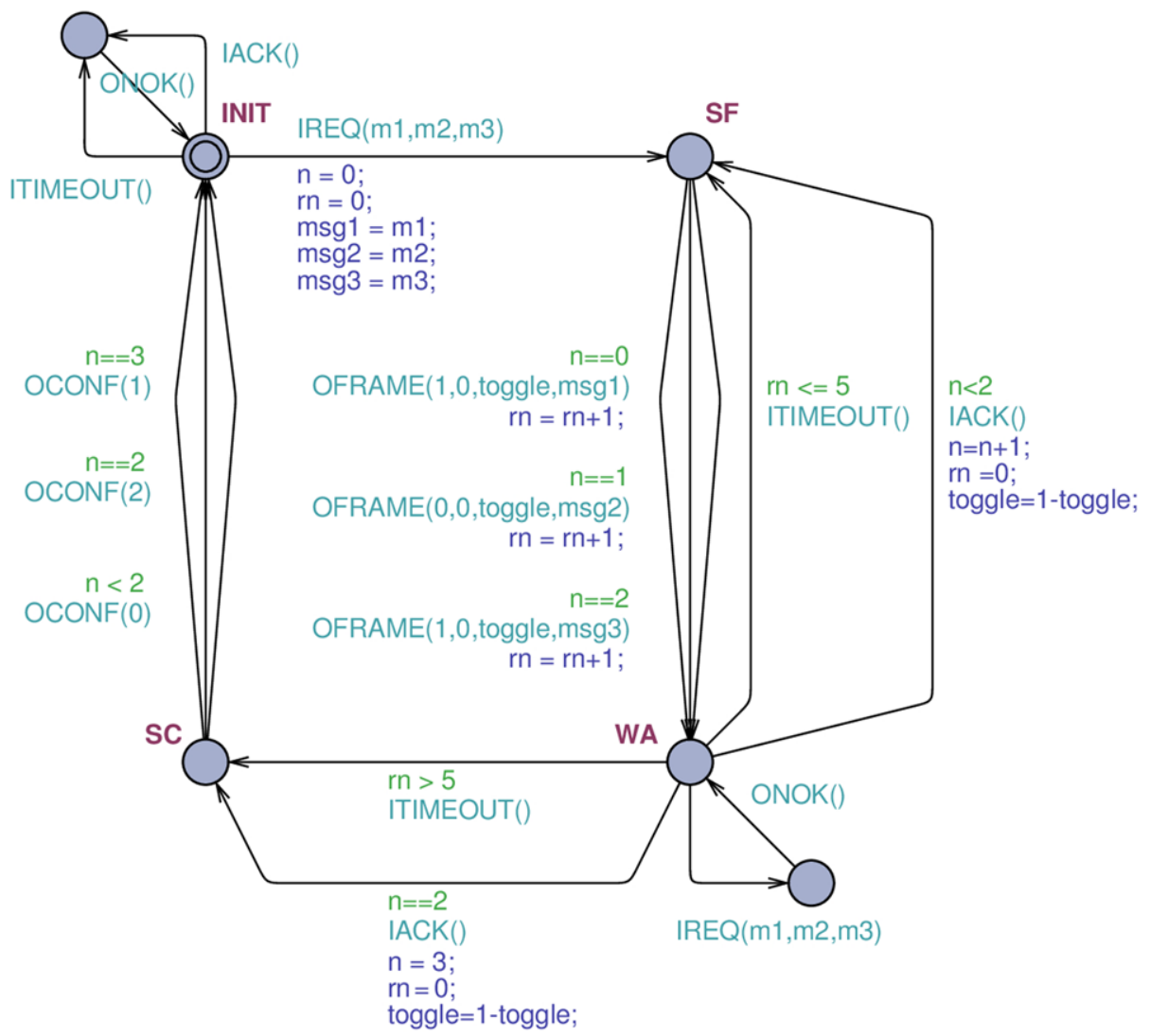

Fig. 10 Mutant 4 


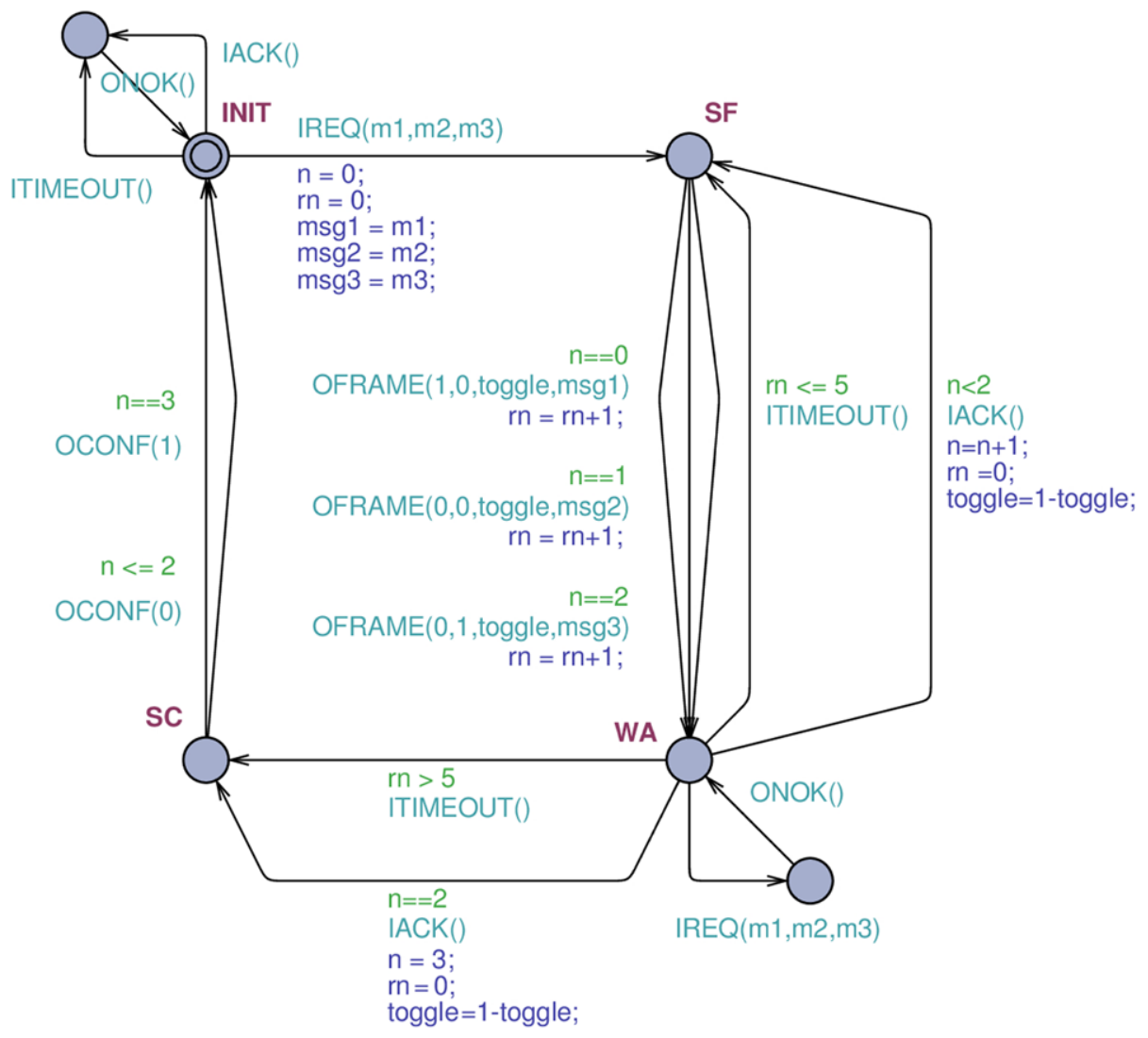

Fig. 11 Mutant 5 


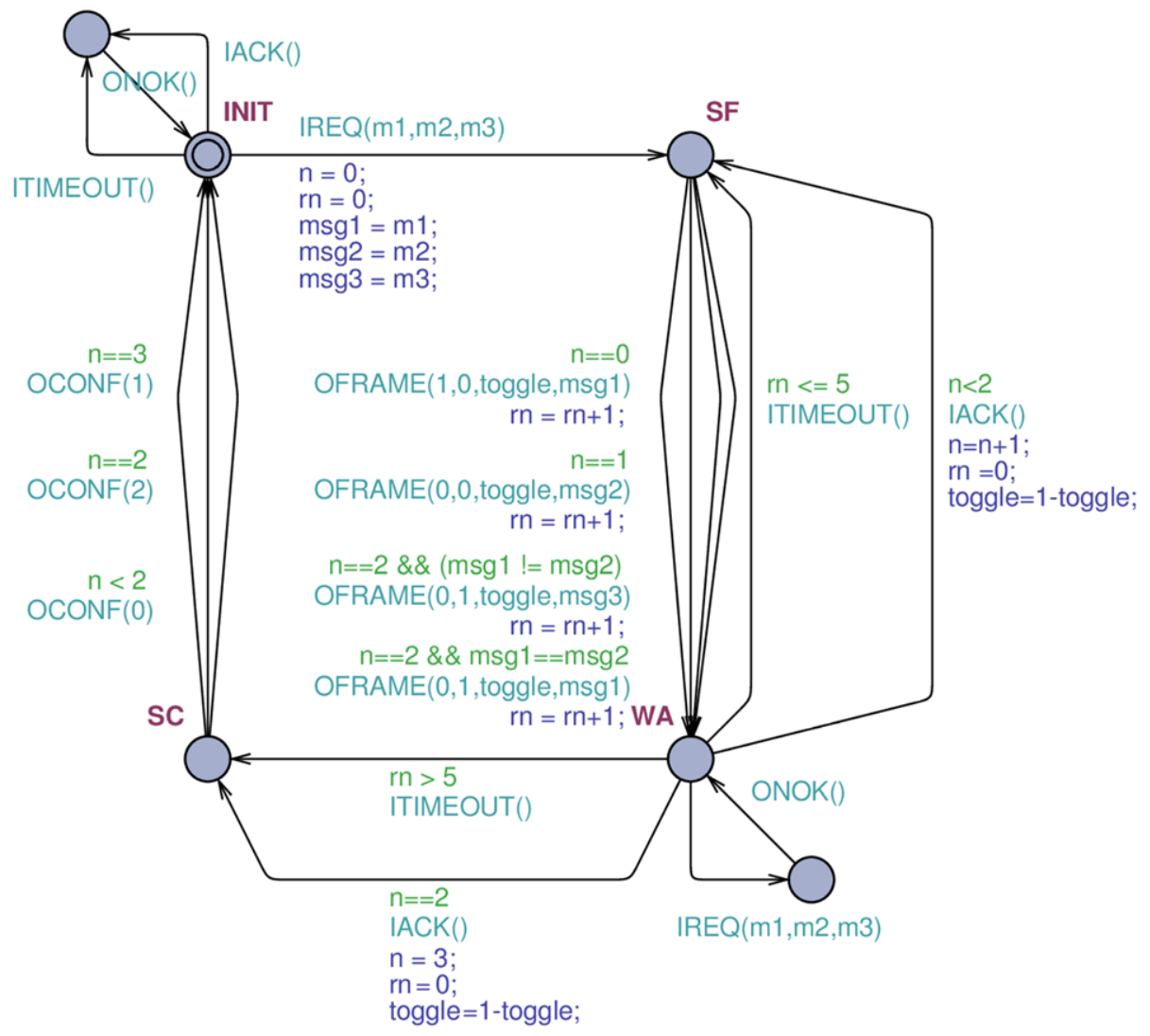

Fig. 12 Mutant 6 


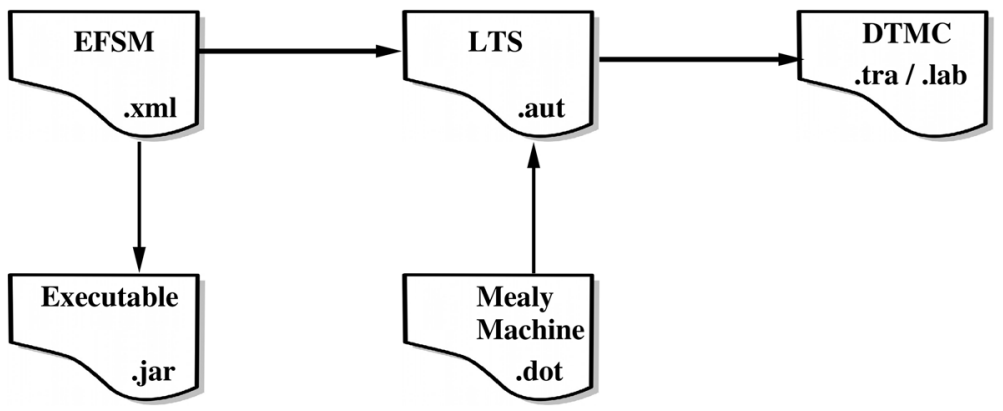

Fig. 13 Formats for representing state machines and implemented conversions

\section{Appendix B: Conversions between input formats}

Figure 13 summarizes the various representations of state machines that we use in this paper, and the conversions between these formats that we have implemented.

The Mealy machine models learned by LearnLib are represented as . dot files. A small script converts Mealy machines in . dot format to Labeled Transition Systems in . aut format by splitting each transition $q \stackrel{i / o}{\longrightarrow} q^{\prime}$ into a pair of two consecutive transitions $q \stackrel{i}{\rightarrow} q^{\prime \prime}$ and $q^{\prime \prime} \stackrel{o}{\rightarrow} q^{\prime}$.

Uppaal models, represented as . xml files, can be translated to the corresponding implementations, encoded as Java . jar files, and to Labeled Transition Systems (LTSs), represented using the .aut format.

We use JTorX to establish conformance of mutant implementations to a model of the reference implementation, represented as an . aut file.

CADP is used in this paper to check strong bisimulation equivalence of labeled transition systems represented as . aut files.

\section{References}

Aarts, F., \& Vaandrager, F. (2010). Learning I/O automata. In Lecture notes in computer science: Vol. 6269. Proceedings of the 21 st international conference on concurrency theory, CONCUR (pp. 71-85). Berlin: Springer.

Aarts, F., Jonsson, B., \& Uijen, J. (2010a). Generating models of infinite-state communication protocols using regular inference with abstraction. In Proceedings of the 22nd IFIP WG 6.1 international conference on testing software and systems, ICTSS'10 (pp. 188-204). Berlin: Springer.

Aarts, F., Schmaltz, J., \& Vaandrager, F. (2010b). Inference and abstraction of the biometric passport. In Proceedings of the 4th international conference on leveraging applications of formal methods, verification, and validation-volume part I (pp. 673-686). Berlin: Springer.

Aarts, F., Heidarian, F., Kuppens, H., Olsen, P., \& Vaandrager, F. (2012a). Automata learning through counterexample-guided abstraction refinement. In Lecture notes in computer science: Vol. 7436. Proceedings of the 18th international symposium on formal methods (FM 2012) (pp. 10-27). Berlin: Springer.

Aarts, F., Kuppens, H., Tretmans, J., Vaandrager, F., \& Verwer, S. (2012b). Learning and testing the bounded retransmission protocol. In JMLR workshop and conference proceedings: Vol. 21. Proceedings of the 11th international conference on grammatical inference (ICGI 2012) (pp. 4-18). JMLR.

Alur, R., \& Dill, D. L. (1994). A theory of timed automata. Theoretical Computer Science, 126, 183-235.

Ammons, G., Bodik, R., \& Larus, J. R. (2002). Mining specifications. In Proceedings of the 29th symposium on principles of programming languages (pp. 4-16). New York: ACM.

Angluin, D. (1987). Learning regular sets from queries and counterexamples. Information and Computation, $75(2), 87-106$ 
Antunes, J., Neves, N., \& Verissimo, P. (2011). Reverse engineering of protocols from network traces. In Proceedings of the working conference on reverse engineering (pp. 169-178).

Aziz, A., Sanwal, K., Singhal, V., \& Brayton, R. (1996). Verifying continuous time Markov chains. In Lecture notes in computer science: Vol. 1102. Proceedings of 8th international conference on computer aided verification (CAV) (pp. 269-276). Berlin: Springer.

Balcázar, J., Diaz, J., Gavaldá, R., \& Watanabe, O. (1997). Algorithms for learning finite automata from queries: a unified view. Advances in Algorithms, Languages, and Complexity, 53-72.

Bartlett, K., Scantlebury, R., \& Wilkinson, P. (1969). A note on reliable full-duplex transmission over halfduplex links. Communications of the ACM, 12, 260-261.

Behrmann, G., David, A., \& Larsen, K. (2004). A tutorial on Uppaal. In Lecture notes in computer science: Vol. 3185. Formal methods for the design of real-time systems (pp. 33-35). Berlin: Springer.

Belinfante, A. (2010). Jtorx: a tool for on-line model-driven test derivation and execution. In Lecture notes in computer science: Vol. 6015. Proceedings of the 16th international conference on tools and algorithms for the construction and analysis of systems (TACAS) (pp. 266-270). Berlin: Springer.

Berg, T., Grinchtein, O., Jonsson, B., Leucker, M., Raffelt, H., \& Steffen, B. (2005). On the correspondence between conformance testing and regular inference. In Lecture notes in computer science: Vol. 3442. Proceedings of the 8th international conference on fundamental approaches to software engineering (FASE) (pp. 175-189). Berlin: Springer.

Bertolino, A., Inverardi, P., Pelliccione, P., \& Tivoli, M. (2009). Automatic synthesis of behavior protocols for composable web-services. In Proceedings of the joint meeting of the 12th European software engineering conference and the 17th ACM SIGSOFT symposium on the foundations of software engineering (pp. 141-150). New York: ACM.

Broy, M., Jonsson, B., Katoen, J.-P., Leucker, M., \& Pretschner, A. (Eds.) (2005). Lecture notes in computer science.: Vol. 3472. Model-based testing of reactive systems. Berlin: Springer.

Castro, J., \& Gavaldà, R. (2008). Towards feasible PAC-learning of probabilistic deterministic finite automata. In Lecture notes in computer science: Vol. 5278. Proceedings of the 9th international colloquium on grammatical inference: algorithms and applications (ICGI) (pp. 163-174). Berlin: Springer.

Cho, C. Y., Babic, D., Shin, E. C. R., \& Song, D. (2010). Inference and analysis of formal models of botnet command and control protocols. In Proceedings of the 17th ACM conference on computer and communications security (pp. 426-439). New York: ACM.

Clark, A., \& Thollard, F. (2004). PAC-learnability of probabilistic deterministic finite state automata. Journal of Machine Learning Research, 473-497.

Clarke, E. (1997). Model checking. In Lecture notes in computer science: Vol. 1346. Proceedings of the 17th conference on foundations of software technology and theoretical computer science (pp. 54-56). Berlin: Springer.

Combe, D., de la Higuera, C., \& Janodet, J.-C. (2010). Zulu: an interactive learning competition. In Lecture notes in computer science: Vol. 6062. Proceedings of the 9th international workshop on finite-state methods and natural language processing (pp. 139-146). Berlin: Springer.

Comparetti, P., Wondracek, G., Kruegel, C., \& Kirda, E. (2009). Prospex: protocol specification extraction. In Proceedings of the 30th IEEE symposium on security and privacy (pp. 110-125). New York: IEEE.

Cook, J. E., \& Wolf, A. L. (1998). Discovering models of software processes from event-based data. ACM Transactions on Software Engineering and Methodology, 7, 215-249.

Cui, W., Kannan, J., \& Wang, H. J. (2007). Discoverer: automatic protocol reverse engineering from network traces. In Proceedings of 16th USENIX security symposium (p. 14).

Dalal, S., Jain, A., Karunanithi, N., Leaton, J., Lott, C., Patton, G., \& Horowitz, B. (1999). Model-based testing in practice. In Proceedings of the 1999 international conference on software engineering, 1999 (pp. 285-294). New York: IEEE.

Dallmeier, V., Lindig, C., Wasylkowski, A., \& Zeller, A. (2006). Mining object behavior with ADABU. In Proceedings of the 2006 international workshop on dynamic systems analysis (WODA) (pp. 17-24). New York: ACM.

D’Argenio, P., Katoen, J.-P., Ruys, T., \& Tretmans, J. (1997). The bounded retransmission protocol must be on time! In Lecture notes in computer science: Vol. 1217. Proceedings of the 3rd workshop on tools and algorithms for the construction and analysis of systems (pp. 416-431). Berlin: Springer.

de la Higuera, C. (2010). Grammatical inference: learning automata and grammars. New York: Cambridge University Press.

de la Higuera, C., \& Janodet, J.-C. (2004). Inference of omega-languages from prefixes. Theoretical Computer Science, 313(2), 295-312.

Denis, F., Lemay, A., \& Terlutte, A. (2000). Learning regular languages using non deterministic finite automata. In Proceedings of the 6th international colloquium on grammatical inference (ICGI) (pp. 3950).

Dijkstra, E. (1969). Notes on structured programming. 
Frantzen, L., Tretmans, J., \& Willemse, T. (2005). Test generation based on symbolic specifications. In Lecture notes in computer science: Vol. 3395. Proceedings of the 5th international workshop on formal approaches to software testing (pp. 1-15). Berlin: Springer.

Garavel, H., Lang, F., Mateescu, R., \& Serwe, W. (2011). CADP 2010: a toolbox for the construction and analysis of distributed processes. In Lecture notes in computer science: Vol. 6605. Proceedings of the 17th international conference on tools and algorithms for the construction and analysis of systems (pp. 372-387). Berlin: Springer.

Gold, E. M. (1978). Complexity of automaton identification from given data. Information and Control, 37(3), 302-320.

Grinchtein, O., Jonsson, B., \& Petterson, P. (2006). Inference of event-recording automata using timed decision trees. In Lecture notes in computer science: Vol. 4137. Proceedings of the 17th international conference on concurrency theory (CONCUR) (pp. 435-449). Berlin: Springer.

Hansson, H., \& Jonsson, B. (1994). A logic for reasoning about time and reliability. Formal Aspects of Computing, 6, 512-535.

Helmink, L., Sellink, M., \& Vaandrager, F. (1994). Proof-checking a data link protocol. In Lecture notes in computer science: Vol. 806. Proceedings international workshop TYPES'93 (pp. 127-165). Berlin: Springer.

Henzinger, T., Nicollin, X., Sifakis, J., \& Yovine, S. (1994). Symbolic model checking for real-time systems. Information and Computation, 111(2), 193-244.

Hungar, H., Niese, O., \& Steffen, B. (2003). Domain-specific optimization in automata learning. In Lecture notes in computer science: Vol. 2725. Proceedings of the 15th international conference on computer aided verification (CAV) (pp. 315-327). Berlin: Springer.

Ip, C., \& Dill, D. (1996). Better verification through symmetry. Formal Methods in System Design, 9(1/2), 41-75.

Katoen, J.-P., Zapreev, I. S., Hahn, E. M., Hermanns, H., \& Jansen, D. N. (2011). The ins and outs of the probabilistic model checker MRMC. Performance Evaluation, 68(2), 90-104.

Kearns, M. J., \& Vazirani, U. V. (1994). An introduction to computational learning theory. Cambridge: MIT Press.

Lee, D., \& Yannakakis, M. (1996). Principles and methods for testing finite state machines—a survey. Proceedings of the IEEE, 84(8), 1090-1123.

Margaria, T., Niese, O., Raffelt, H., \& Steffen, B. (2004). Efficient test-based model generation for legacy reactive systems. In Proceedings of the 9th IEEE international high-level design validation and test workshop (HLDVT) (pp. 95-100). Washington: IEEE Computer Society.

Mariani, L., Pastore, F., \& Pezze, M. (2011). Dynamic analysis for diagnosing integration faults. IEEE Transactions on Software Engineering, 37, 486-508.

Meinke, K., \& Walkinshaw, N. (2012). Model-based testing and model inference. In Lecture notes in computer science: Vol. 7609. Proceedings of the 5th international symposium on leveraging applications of formal methods, verification and validation (ISoLA) (pp. 440-443). Berlin: Springer.

Mostowski, W., Poll, E., Schmaltz, J., Tretmans, J., \& Wichers Schreur, R. (2009). Model-based testing of electronic passports. In Lecture notes in computer science: Vol. 5825. Proceedings of the 15th international workshop on formal methods for industrial critical systems (pp. 207-209). Berlin: Springer.

Puterman, M. L. (1994). Markov decision processes: discrete stochastic dynamic programming (1st ed.). New York: Wiley.

Raffelt, H., Steffen, B., Berg, T., \& Margaria, T. (2009). Learnlib: a framework for extrapolating behavioral models. International Journal on Software Tools for Technology Transfer, 11, 393-407.

Settles, B. (2010). Active learning literature survey (Technical report). University of Wisconsin-Madison.

Shafique, M., \& Labiche, Y. (2010). A systematic review of model based testing tool support (Technical Report SCE-10-04). Department of Systems and Computer Engineering, Carleton University, Ottawa, Canada.

Sudkamp, T. A. (2006). Languages and machines: an introduction to the theory of computer science (3rd ed.). Reading: Addison-Wesley.

Tretmans, J. (2008). Model based testing with labelled transition systems. In Lecture notes in computer science: Vol. 4949. Formal methods and testing (pp. 1-38). Berlin: Springer.

Tretmans, J., \& Brinksma, E. (2003). Torx: automated model-based testing. In Proceedings of the 1st European conference on model-driven software engineering (pp. 31-43).

Utting, M., \& Legeard, B. (2007). Practical model-based testing: a tools approach. San Mateo: MorganKaufmann.

van der Aalst, W. M. P. (2011). Process mining-discovery, conformance and enhancement of business processes. Berlin: Springer.

Verwer, S. (2010). Efficient identification of timed automata: theory and practice. PhD thesis, Delft University of Technology. 
Verwer, S., de Weerdt, M., \& Witteveen, C. (2011). Efficiently identifying deterministic real-time automata from labeled data. Machine Learning, 1-39.

Walkinshaw, N., Bogdanov, K., Holcombe, M., \& Salahuddin, S. (2007). Reverse engineering state machines by interactive grammar inference. In Proceedings of the 14th working conference on reverse engineering (pp. 209-218). New York: IEEE.

Walkinshaw, N., Bogdanov, K., Damas, C., Lambeau, B., \& Dupont, P. (2010a). A framework for the competitive evaluation of model inference techniques. In Proceedings of the 1st international workshop on model inference in testing (pp. 1-9). New York: ACM.

Walkinshaw, N., Bogdanov, K., Derrick, J., \& Paris, J. (2010b). Increasing functional coverage by inductive testing: a case study. In Lecture notes in computer science: Vol. 6435. Proceedings of the 22nd IFIP WG 6.1 international conference on testing software and systems (ICTSS) (pp. 126-141). Berlin: Springer.

Weyuker, E. J. (1983). Assessing test data adequacy through program inference. ACM Transactions on Programming Languages and Systems, 5(4), 641-655.

Yokomori, T. (1993). Learning non-deterministic finite automata from queries and counterexamples. In $M a-$ chine Intelligence (pp. 196-189). University Press. 